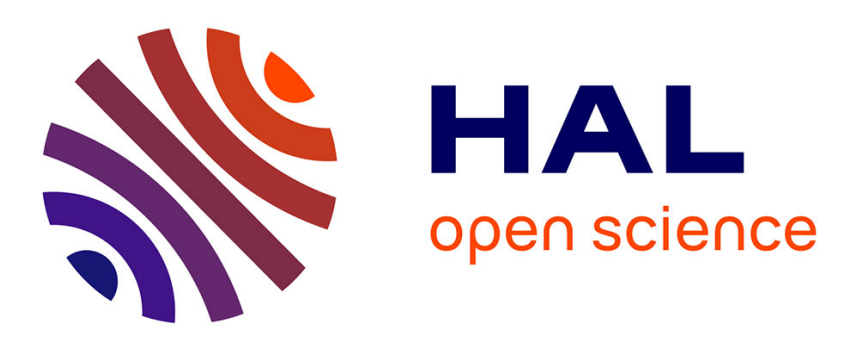

\title{
A simplified approach for the calculation of acoustic emission in the case of friction-induced noise and vibration
}

\author{
Kevin Soobbarayen, Sébastien Besset, Jean-Jacques Sinou
}

\section{To cite this version:}

Kevin Soobbarayen, Sébastien Besset, Jean-Jacques Sinou. A simplified approach for the calculation of acoustic emission in the case of friction-induced noise and vibration. Mechanical Systems and Signal Processing, 2015, 50-51, pp.732-756. 10.1016/j.ymssp.2014.05.014 . hal-01009235

\section{HAL Id: hal-01009235 https://hal.science/hal-01009235}

Submitted on 17 Jun 2014

HAL is a multi-disciplinary open access archive for the deposit and dissemination of scientific research documents, whether they are published or not. The documents may come from teaching and research institutions in France or abroad, or from public or private research centers.
L'archive ouverte pluridisciplinaire HAL, est destinée au dépôt et à la diffusion de documents scientifiques de niveau recherche, publiés ou non, émanant des établissements d'enseignement et de recherche français ou étrangers, des laboratoires publics ou privés. 


\title{
A simplified approach for the calculation of acoustic emission in the case of friction-induced noise and vibration
}

\author{
K. Soobbarayen ${ }^{1, a}$, S. Besset ${ }^{1, b}$, and J-.J. Sinou ${ }^{1, c}$ \\ ${ }^{1}$ Laboratoire de Tribologie et Dynamique des Systèmes, UMR CNRS 5513, \\ Ecole Centrale de Lyon, 36 avenue Guy de Collongue 69134 Ecully Cedex, France \\ \{ ${ }^{\mathrm{a}}$ kevin.soobbarayen, ${ }^{\mathrm{b}}$ sebastien.besset, ${ }^{\mathrm{c}}$ jean-jacques.sinou\}@ec-lyon.fr
}

\begin{abstract}
The acoustic response associated with squeal noise radiations is a hard issue due to the need to consider non-linearities of contact and friction, to solve the associated nonlinear dynamic problem and to calculate the noise emissions due to self-excited vibrations. In this work, the focus is on the calculation of the sound pressure in free space generated during squeal events.

The calculation of the sound pressure can be performed by the Boundary Element Method (BEM). The inputs of this method are a boundary element model, a field of normal velocity characterized by a unique frequency. However, the field of velocity associated with friction-induced vibrations is composed of several harmonic components. So, the BEM equation has to be solved for each frequencies and in most cases, the number of harmonic component is significant. Therefore, the computation time can be prohibitive.

The reduction of the number of harmonic component is a key point for the quick estimation of the squeal noise. The proposed approach is based on the detection and the selection of the predominant harmonic components in the mean square velocity. It is applied on two cases of squeal and allows us to consider only few frequencies.

In this study, a new method will be proposed in order to quickly well estimate the noise emission in free space. This approach will be based on an approximated acoustic power of brake system which is assumed to be a punctual source, an interpolated directivity and the decrease of the acoustic power levels.

This method is applied on two classical cases of squeal with one and two unstable modes. It allows us to well reconstruct the acoustic power levels map. Several error estimators are introduced and show that the reconstructed field is close to the reference calculated with a complete BEM.
\end{abstract}

\section{Introduction}

The brake squeal phenomenon is characterized by high frequency noise emissions due to frictioninduced self-excited vibrations. The prediction and the calculation of squeal noise are complex tasks which are composed of several steps [1]. The first one deals with the nonlinear modeling with the definition of the mechanical system geometry, the introduction of nonlinear laws corresponding to the contact and friction phenomenon over the interface. Then, a stability analysis is performed with respect to one or several parameters. This analysis allows us to detect the unstable equilibrium configurations that may lead to squeal and provides the fundamental frequencies of the unstable modes. The next step consists of calculating the time responses and it has been shown in the literature that the stationary regime associated with the squeal has a spectrum composed of fundamental frequencies (that should be different from the frequencies of 
the unstable modes [2]), their harmonic components and also their linear combinations. Then, the calculation of the noise radiations during squeal event can be performed with the Boundary Element Method [3,4]. This is the classical way to well estimate the sound pressure. This numerical method is based on the resolution of the Kirchhoff-Helmholtz equation which depends on the wave frequency. Therefore, the sound pressure calculation has to be carried out for each harmonic component of the velocity field.

In this aim, the multi-frequency acoustic calculation method has previously been developed [1]. The method is based on the decomposition of the velocity into Fourier series. Therefore, the global wave is decomposed into elementary waves with a unique frequency. The BEM is then applied for each wave and the global sound pressure field is calculated by superposition. The application of the BEM is composed of three steps. Firstly the boundary element model is built and it is composed of the system skin. Secondly, the surface sound pressure is calculated and finally, the sound pressure in the free space is evaluated by using the surface pressure. So, the latter is calculated for each frequency and the free field pressure is calculated for each frequency and each field plane. However, the number of frequencies can be significant making the computation time prohibitive.

In a previous work [1], it has been shown that only few harmonic components are predominant in the acoustic response. So it can be useful to determine these frequencies before the application of the multi-frequency acoustic calculation method. The first aim of the current paper is to develop a criterion based on the mean square velocity convergence (i.e. the dynamic response) which allows us to detect the predominant frequencies.

The second objective of this work is to propose a new method which allows to quickly well estimate the sound pressure in the free field. In order to calculate the radiations, the directivity and a propagation model which describe the level decrease are required. The main idea is to determine the directivity with the BEM and to determine an analytical function with an interpolation. Finally, for each frequencies, the sound pressure can be evaluated at every field points without the BEM.

The present paper is organized as follows. Firstly, the vibroacoustic of a squealing disc brake system is presented. The brake system under study is detailed and the dynamic and acoustic responses for two cases with one and two unstable modes are given. Secondly, the criterion which allows us to detect the predominant frequencies in the dynamic response is presented and applied for the two cases under study. Thirdly, the acoustic approximation method is presented and several sound pressure error estimators are presented. Finally, the acoustic method is applied and validated on the two cases.

\section{Vibroacoustic of a squealing brake system}

In this section, the simplified disc brake model under study is presented. The finite element model, the modeling of the frictional interface and the stability results are detailed. Next, the focus will be on the time responses associated with two classical cases of squeal with one and two unstable modes. Finally, the multi-frequency acoustic calculation method is applied to calculate the surface sound pressure and the sound pressure radiated in free space.

\subsection{Brake system modeling}

The two main components involved in the squeal are the disc and the pad which share a frictional interface. This allows to focus on a simplified disc brake system composed of a circular disc and a pad as illustrated in Figure 1. The inner radius of the disc is clamped due to the shaft connection and the outline of the upper surface of the pad can only translate along the normal 


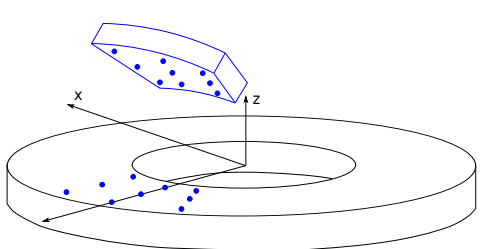

(a)

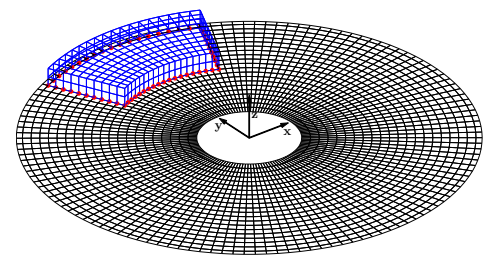

(b)

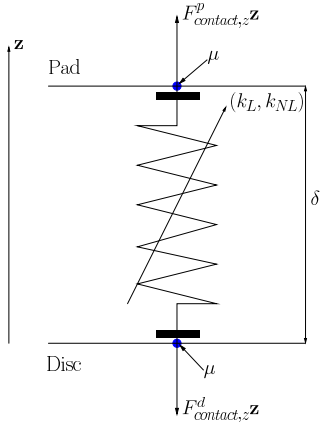

(c)

Figure 1: Brake system model and boundary element model. (a): Simplified disc brake system and selected contact nodes $\bullet$; (b): boundary element mesh used for the acoustic calculation.

$\mathbf{z}$-direction to represent the caliper connection. In this work, automotive brake dimensions have been used.

Contact and friction phenomenon occurs over the common frictional interface which is modeled with nine uniformly spaced contact points as illustrated in Figure 1 (a). These contact elements are arbitrarily chosen and these number and positions strongly influence the stability analysis. However, increasing the number provides a better description of the interface but the calculation performances can be much reduced. In this study, nine elements seem to be a good compromise [2].

Between the previous selected points, nonlinear contact and friction forces are introduced. The nonlinear contact force vector is described with linear and nonlinear stiffnesses and contact/loss of contact configurations is considered. The cubic contact law associated with nonlinear contact elements takes the following expression:

$$
F_{\text {contact }, z}^{\mathrm{d}}=\left\{\begin{array}{l}
k_{\mathrm{L}} \delta+k_{\mathrm{NL}} \delta^{3} \text { if } \delta<0 \\
0 \text { otherwise }
\end{array}\right.
$$

where $\delta=X_{\mathrm{p}}-X_{\mathrm{d}}$ is the relative displacement, $X_{\mathrm{p}}$ and $X_{\mathrm{d}}$ denote the normal displacements of the pad and the disc respectively. $k_{\mathrm{L}}$ and $k_{\mathrm{NL}}$ are the linear and cubic stiffnesses, $F_{\text {contact }, z}^{\mathrm{p}}$ and $F_{\text {contact }, z}^{\mathrm{d}}$ are the components of the normal contact force vector applied to the pad and the disc respectively. It can be noted that $F_{\text {contact, } z}^{\mathrm{p}}=-F_{\text {contact, } z}^{\mathrm{d}}$. This contact force expression has been chosen to fit experiments as explained in [5]. The main limitation of this formulation is that it allows penetration between the disc and the pad due to the fact that the contact stiffnesses are constant. A penalty algorithm which adjusts these stiffnesses can be used to apply an impenetrability condition.

Involving friction, the friction coefficient $\mu$ is assumed to be constant for the sake of simplicity and the classical Coulomb's law is used to model the friction over the interface. Thus, the nonlinear friction force vector over the frictional interface plane is defined by:

$$
\left\{\begin{array}{l}
\mathbf{F}_{\text {friction }}^{\mathrm{d}}=\mu F_{\text {contact }, z}^{\mathrm{d}} \frac{\mathbf{v}_{\mathrm{r}}}{\left\|\mathbf{v}_{\mathrm{r}}\right\|} \\
\mathbf{F}_{\text {friction }}^{\mathrm{p}}=-\mathbf{F}_{\text {friction }}^{\mathrm{d}}
\end{array}\right.
$$

where $\mathbf{F}_{\text {friction }}^{\mathrm{d}}$ and $\mathbf{F}_{\text {friction }}^{\mathrm{d}}$ are the friction force vectors applied to the disc and the pad respectively. $\mathbf{v}_{\mathrm{r}}$ is the sliding velocity between the disc and the pad. A permanent sliding state is considered. Finally, the braking pressure which activates the contact between the disc and the pad is modeled with a constant uniform pressure applied over the back-plate of the pad. 


\begin{tabular}{ccccc}
\hline Case & Friction coefficient & Frequency $f_{1} \mathrm{~Hz}$ & Frequency $f_{2} \mathrm{~Hz}$ & Expected spectrum \\
\hline 1 & 0.72 & 929.8 & - & $p f_{1}, p \in \mathbb{N}^{*}$ \\
2 & 0.74 & 930.3 & 9418 & $\pm m f_{1} \pm n f_{2}, m, n \in \mathbb{N}$ \\
\hline
\end{tabular}

Table 1: List of cases under study. (1): One unstable mode; (2): two unstable modes.

This simplified modeling does not attempt to capture all the nonlinear effects but is suitable for mode coupling instabilities. Moreover, it serves to illustrate the simplified approach for the calculation of acoustic emission in the case of friction-induced noise and vibration.

A Craig and Bampton reduction method [6] is used to reduce the system size. The reduction basis is composed of all the attachment modes and the first hundred eigenmodes of the structure assuming the interface nodes are held fixed. This reduction provides a good correlation between the whole and the reduced brake models until $20 \mathrm{kHz}$ which is sufficient for the frequency range of interest of the present study.

Finally, the equations of motion in the reduction space are given by Eq. 3:

$$
\mathbf{M} \ddot{\mathbf{X}}+\mathbf{C} \dot{\mathbf{X}}+\mathbf{K X}=\mathbf{F}_{\mathrm{NL}}(\mathbf{X})+\mathbf{F}
$$

where $\mathbf{M}, \mathbf{C}$ and $\mathbf{K}$ are mass, damping and stiffness matrices. $\mathbf{X}$ is the generalized displacement vector and the dot denotes derivative with respect to the time. $\mathbf{F}_{\mathrm{NL}}$ defines the global nonlinear force vector which contains linear and nonlinear parts of the contact force vector and also the friction force vector applied to the disc and the pad. The vector $\mathbf{F}$ corresponds to the braking pressure applied over the pad back-plate. Involving the damping matrix $\mathbf{C}$, the following modal damping is considered: a damping percentage $\xi$ is used for stable modes and a damping rate $\zeta_{i}$ for the $i$ th coalescent modes, as explained in [1].

For the interested reader, extensive reviews about the modeling of the automotive disc brake squeal can be found in $[7,8]$.

\subsection{Stability analysis}

The stability can now be estimated by analyzing the eigenvalues of the linearized equation of motion. The linearization is performed near the sliding equilibrium configuration $\mathbf{X}_{\text {sliding }}$ which corresponds to the quasi-static configuration of the rotating brake system under the braking pressure. The eigenvalues and the eigenvectors of the linearized system are given by the following equation:

$$
\left(\lambda^{2} \mathbf{M}+\lambda \mathbf{C}+\left(\mathbf{K}-\mathbf{J}_{\mathrm{NL}, \mathbf{X}_{\text {sliding }}}\right)\right) \boldsymbol{\Phi}=\mathbf{0}
$$

where $\boldsymbol{\lambda}$ is the diagonal matrix which contains the eigenvalues, $\boldsymbol{\Phi}$ denotes the eigenvector matrix, and $\mathbf{J}_{\mathrm{NL}, \mathbf{X}_{\text {sliding }}}$ is the Jacobian of the nonlinear force vector. The eigenvalues are complex due to friction and if all the eigenvalues have a negative real part, the sliding equilibrium is stable and the system reaches the sliding equilibrium configuration $\mathbf{X}_{\text {sliding. }}$. If at least one real part is positive, then the sliding equilibrium is unstable, the system diverges and self-excited vibrations are generated. In this work, this analysis is carried out with respect to the friction coefficient $\mu$. By analyzing the eigenvalues with respect to $\mu$, two instabilities with one and two unstable modes are detected. The two cases under study in this work are listed in Table 1.

The stability analysis, based on the associated linear system, allows to predict the squeal onset and this ability has been experimentally validated by Massi et al. [9]. However, the authors highlight the fact that a nonlinear model needs to be used to reproduce the squeal in the time 
domain. That is why a full time integration of the nonlinear system has to be performed to calculate the nonlinear friction-induced squeal vibrations.

\subsection{Self-excited vibrations}

The time responses are calculated with a Runge-Kutta integration scheme for the cases 1 and 2. The initial conditions correspond to the associated sliding equilibrium configurations with a slight disturbance. The results are similar with those shown in [1] and in order to condense the contents of the present paper, only the spectrum of the stationary regimes for the two cases is given.

For the case 1, Figure 2 (a) indicates that the harmonic components are composed of the fundamental frequency $f_{1}$ and several harmonic as $2 f_{1}$ and $3 f_{1}$.

The case 2 presents a more complex spectrum composed of the fundamental frequencies $f_{1}$ and $f_{2}$, several harmonic components $2 f_{1}, 3 f_{1}, 2 f_{2}$, and also their linear combinations of the form: $\pm m f_{1} \pm n f_{2}$ where $m$ and $n$ are positive integers (see Figure 2 (b)).

These dynamics responses strongly depend on the contact status due to successive activation/deactivation of the contact stiffnesses during contact/loss of contact configurations. The interface motion is therefore complex and the interested reader could find more details about the contact status during squeal event in [2].

\subsection{Noise emissions}

The numerical study of acoustic radiations due to squeal events is a recent topic. In 2004, an approach using a semi analytical plate kinematic has been used to estimate the noise radiations due to modal force vibrations [10]. More recently, Oberst et al. [11] propose complete guidelines for the numerical acoustic investigation of squeal. This study highlights the fact that the pad geometry, the interface refinement and the contact/friction formulation have a strong impact on the noise radiations in terms of both the levels and the directivity. The two mentioned papers focus on the radiations generated with a forced dynamic response for a unique frequency. With this method, it is possible to well estimate the radiations associated with a given mode. In the present paper, the proposed approach involves the global radiations generated by the whole dynamic responses i.e. with the whole nonlinear spectrum for a simplified phenomenological brake model.

For this acoustic study, the boundary element model is composed of the upper part of the system skin as illustrated in Figure 1 (b). Actually, it is assumed that these surface radiations lead the outside global noise radiations. Moreover, over the connected points between the disc and the pad (i.e. the red points in Figure 1 (a)), the normal velocities are averaged. Then the multi-frequency acoustic calculation method allows us to estimate the noise levels over the mesh and at every points in free space. The main idea of this approach is to apply the BEM for each frequency and to calculate the global sound pressure by superposition. This method has been developed in a previous work and the interested reader could refer to [1]. The BEM contains two main steps: firstly the surface sound pressure calculation $\mathbf{P}_{S}^{\omega}$ which depends on the surface normal velocity field $\dot{\mathbf{X}}_{\mathrm{n}}$, then the free space sound pressure $\mathbf{P}^{\omega}$ is calculated with the surface sound pressure. For more details about the Boundary Element Method, the reader could refer to $[3,4]$. The previous fields depend on the wave frequency $\omega$, that is why the BEM has to be applied for each harmonic component. The global sound pressure field is calculated by superposition and will be denoted by $\mathbf{P}$ in the free space and $\mathbf{P}_{\mathbf{S}}$ over the mesh. For the two cases under study, the focus will be on the sound pressure levels $L_{\mathrm{p}, \text { bem }}=10 \log _{10}\left(\mathbf{P P}^{*} / P_{\text {ref }}^{2}\right)$, where $P_{\text {ref }}=20 \mu \mathrm{Pa}$ and the star denotes the conjugated. 


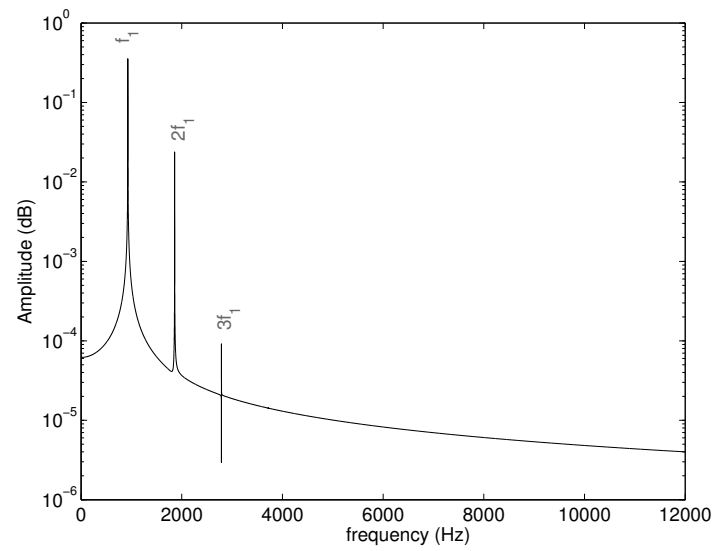

(a)

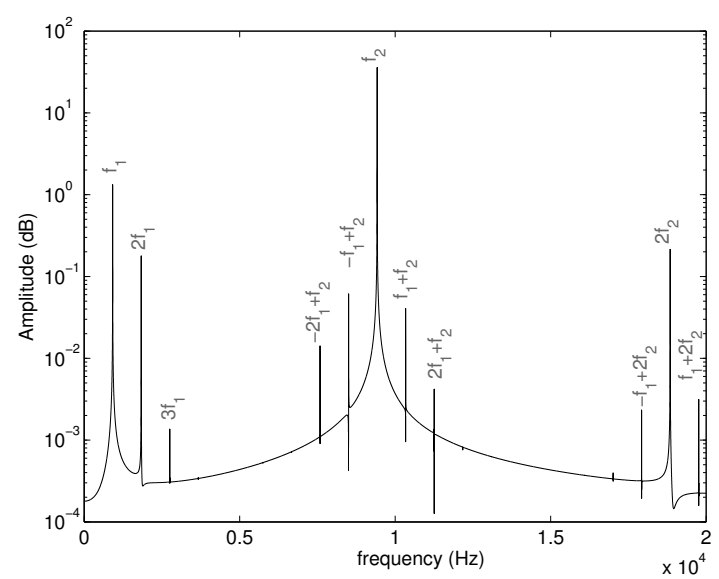

(c)

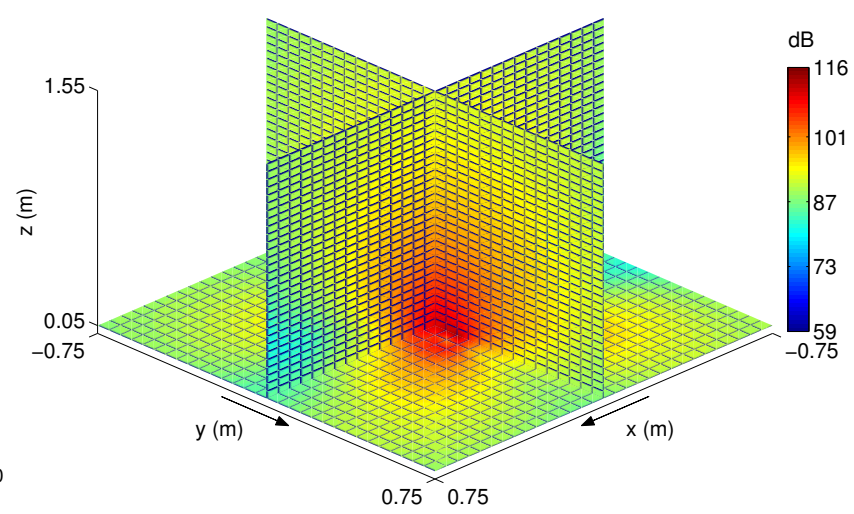

(b)

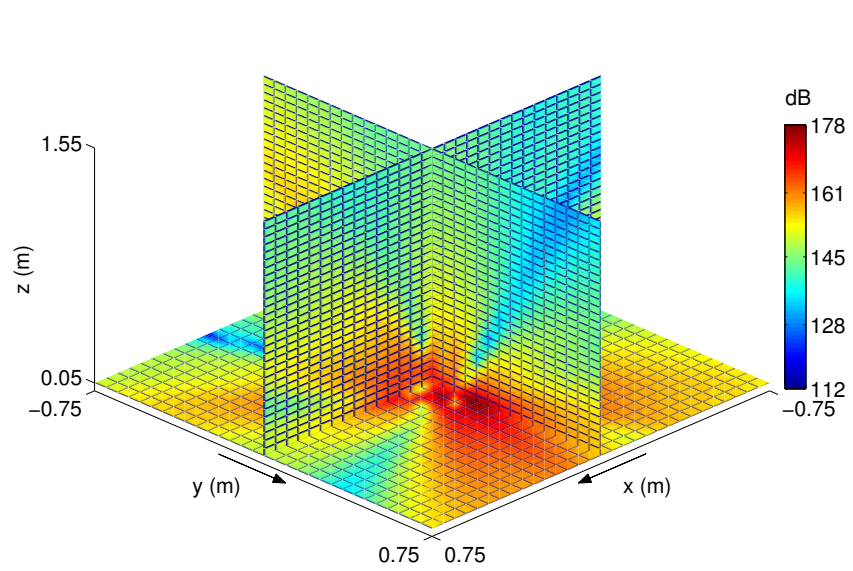

(d)

Figure 2: Nonlinear spectra and sound pressure levels in free space for the cases 1 and 2. (a): Spectrum case 1; (b): $L_{p, \text { bem }}$ case 1; (c): spectrum case 2; (d): $L_{p, \text { bem }}$ case 2.

For the case 1, the total sound pressure levels radiated in free space are composed of one main lobe along the $\mathbf{z}$-direction, but four lobes are present over the $(\mathbf{x}, \mathbf{y})$ plane as indicated in Figure 2 (c).

For the case 2, the free space radiations are more complex with several main directions of propagation as illustrated in Figure 2 (d). This is due to the contribution of two unstable modes in the dynamic response. Moreover, noise levels are also higher than for the case 1 due to the higher amplitude of velocity.

It can be concluded that both the dynamic and acoustic responses are much complex for the multi-instability case: the directivity presents several main propagation directions and higher noise levels. The computation time of the surface pressure can be prohibitive due to the mesh refinement and the frequency dependency. However, it can be noticed that the total surface pressure is widely led by only few harmonic components. This feature has been investigated in details in [1] where a convergence study of the sound pressure is carried out with respect to the number of retained harmonic components. Therefore, if a criterion can be determined to select the predominant harmonic components before solving the acoustic problem, it will be possible 
to quickly well estimate the sound pressure.

These numerical results are not experimentally confirmed but the main objective of the present study is to propose a harmonic components selection method and an acoustic approximation method. So, the development and the validation of the proposed simplified approach are valid. However, squeal events with one or two unstable modes at the same time have been experimentally reported in $[12,13]$ respectively.

\section{Harmonic components selection method}

As explained in the previous section, the dynamic response associated with the squeal is composed of several frequencies. These harmonic components can be numerous as for the case with two unstable modes and the sound pressure has to be calculated for each frequency. However, as previously explained, the total radiation is mainly led by few harmonic components. In this section, a criterion which allows the detection of the predominant frequencies is presented. The main idea is to select the harmonic components which "significantly" contribute to the mean square surface normal velocity. By this way, an optimal Fourier basis is built with a limited number of harmonic components.

\subsection{Mean square velocity convergence}

The initial Fourier basis is composed of all the detected harmonic components sorted by increasing order. The detected components are linear combinations of the fundamental frequencies and are of the form:

$$
k_{1} w_{1}+k_{2} w_{2}+\ldots+k_{i} w_{i}+\ldots+k_{p} w_{p}
$$

with $k_{i} \in\left[-N_{\mathrm{h}}, N_{\mathrm{h}}\right], N_{\mathrm{h}}$ is the highest order and $p$ denotes the number of detected fundamental frequencies. Fourier transform of the surface normal velocity field provides the following expression:

$$
\dot{\mathbf{X}}_{\mathrm{n}}(t) \approx \sum_{k_{1}=-N_{\mathrm{h}}}^{N_{\mathrm{h}}} \ldots \sum_{k_{p}=-N_{\mathrm{h}}}^{N_{\mathrm{h}}} \mathbf{a}_{k_{1}, \ldots, k_{p}} \cos \left(k_{1} w_{1}+\ldots+k_{p} w_{p}\right) t+\mathbf{b}_{k_{1}, \ldots, k_{p}} \sin \left(k_{1} w_{1}+\ldots+k_{p} w_{p}\right) t
$$

where $\mathbf{a}_{k_{1}, \ldots, k_{p}}$ and $\mathbf{b}_{k_{1}, \ldots, k_{p}}$ are the Fourier coefficient vectors corresponding to the linear combination of $w_{1}, \ldots, w_{p}$. By introducing the basis $\boldsymbol{\omega}=\left[\begin{array}{lll}w_{1} & \ldots & w_{p}\end{array}\right]^{T}$ and the vector $\boldsymbol{\tau}=\boldsymbol{\omega} t$, the approximated velocity takes the following form:

$$
\dot{\mathbf{X}}_{\mathrm{n}}(\boldsymbol{\tau}) \approx \mathbf{a}_{0}+\sum_{\mathbf{k} \in \mathbb{Z}^{p}} \mathbf{a}_{\mathrm{k}} \cos (\mathbf{k} \cdot \boldsymbol{\tau})+\mathbf{b}_{\mathrm{k}} \sin (\mathbf{k} \cdot \boldsymbol{\tau})
$$

where the vector $\mathbf{k}$ contains the coefficients of all the linear combinations of the fundamental pulsations $\omega_{j}$. It can be noticed that the velocity associated with the harmonic component $m$ is of the following form:

$$
\dot{\mathbf{X}}_{\mathrm{n}, m}(\boldsymbol{\tau})=\mathbf{a}_{\mathrm{k}_{\mathrm{m}}} \cos \left(\mathbf{k}_{m} \cdot \boldsymbol{\tau}\right)+\mathbf{b}_{\mathrm{k}_{\mathrm{m}}} \sin \left(\mathbf{k}_{m} \cdot \boldsymbol{\tau}\right)
$$

The mean square velocity associated with a truncation $N$ and denoted by $E_{\mathrm{k}, N}$ can now be calculated:

$$
E_{\mathrm{k}, N}=\sqrt{\frac{1}{N_{\mathrm{dof}}}\left[\mathbf{a}_{0}+\sum_{m=1}^{N} \dot{\mathbf{X}}_{\mathrm{n}, m}(\boldsymbol{\tau})\right]^{T}\left[\mathbf{a}_{0}+\sum_{m=1}^{N} \dot{\mathbf{X}}_{\mathrm{n}, m}(\boldsymbol{\tau})\right]}
$$


where $N$ denotes the current truncation and $N_{\text {dof }}$ is the number of degree of freedom of the acoustic model. Then, the mean square velocity associated with the global response $E_{\mathrm{k}, \text { ref }}$ can be calculated and used as a reference:

$$
E_{\mathrm{k}, \mathrm{ref}}=E_{\mathrm{k}, N_{\max }}
$$

where $N_{\max }$ denotes the number of harmonic components detected in the dynamic response. Finally, the relative error $\epsilon_{\mathrm{k}, N}$ is introduced to evaluate the convergence of the mean square velocity with respect to the number of retained harmonic components:

$$
\epsilon_{\mathrm{k}, N}=\left\|\frac{E_{\mathrm{k}, N}-E_{\mathrm{k}, \mathrm{ref}}}{E_{\mathrm{k}, \mathrm{ref}}}\right\| \text { with } N=1 \ldots N_{\max }
$$

\subsection{Building of the optimized Fourier basis}

The analysis of the previous error $\epsilon_{\mathrm{k}, N}$ allows the detection of the predominant harmonic components. The first step of this analysis is to introduce a target error Tole1. Then, the harmonic components which "significantly" improve the mean square velocity are detected. As illustrated in Figure 3, all the gaps $d_{i}=\left|\epsilon_{\mathrm{k}, i+1}-\epsilon_{\mathrm{k}, i}\right|$ are calculated and the highest correspond to the predominant components (i.e. by introducing a threshold Tole2). The optimized Fourier basis is built by retaining only these predominant components. Then, the mean square velocity associated with the optimized Fourier basis $E_{\mathrm{k}, \mathrm{opt}}$ is calculated:

$$
E_{\mathrm{k}, \mathrm{opt}}=\sum_{m \in \mathbb{P}} E_{\mathrm{k}, m}
$$

where $\mathbb{P}$ denotes the current optimal basis defined by:

$$
\mathbb{P}=\left\{i \in\left[1 ; N_{\max }\right] / d_{i}<\text { Tole } 2\right\}
$$

$E_{\mathrm{k}, \mathrm{opt}}$ is compared with the reference mean square velocity $E_{\mathrm{k}, \text { ref }}$ with the following relative error:

$$
\epsilon_{\mathrm{k}, \mathrm{opt}}=\left\|\frac{E_{\mathrm{k}, \mathrm{opt}}-E_{\mathrm{k}, \mathrm{ref}}}{E_{\mathrm{k}, \mathrm{ref}}}\right\|
$$

If the target Tole1 is not reached, the "less" predominant components associated with the "lower" gaps $d_{i}$ are added in the Fourier basis and $\mathbb{P}$ is updated. The final optimal Fourier basis $\mathbb{P}_{\text {opt }}$ corresponds to the following set:

$$
\mathbb{P}_{\text {opt }}=\left\{i \in\left[1 ; N_{\text {max }}\right] / d_{i}<\text { Tole } 2, \epsilon_{\mathrm{k}, \mathrm{opt}}<\text { Tole } 1\right\}
$$

This method is illustrated in Figure 4.

\subsection{Relevance of the optimized Fourier basis}

In order to evaluate the relevance of the optimized Fourier basis, the surface acoustic power error is also analyzed. This analysis is conducted by performing the complete direct BEM calculation and is just presented to justify the use of the optimized basis. The main idea is to calculate the relative error between the optimized surface acoustic power and the reference calculated with all the harmonic components.

The surface acoustic power associated with the frequency $\omega_{i}$ is of the following form:

$$
W_{S}\left(\omega_{i}\right)=\frac{1}{2} \Re\left[\mathbf{P}_{S}\left(\omega_{i}\right) \dot{\mathbf{X}}_{\mathrm{n}, i}^{*}\right]
$$




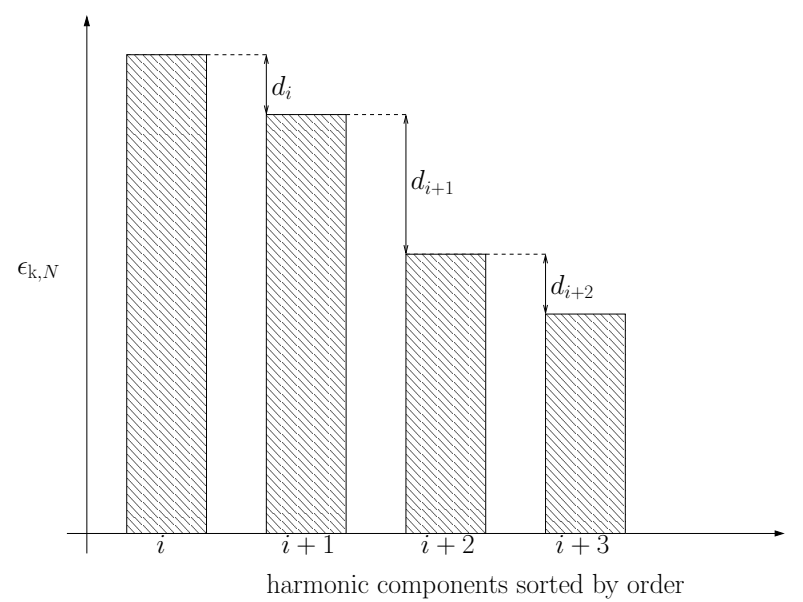

Figure 3: Detection of the predominant harmonic component

The surface acoustic power associated with the truncation $N$ is given by:

$$
W_{N}=\sum_{i=1}^{N} W_{S}\left(\omega_{i}\right)
$$

The reference acoustic power $W_{\text {ref }}$ is calculated with all the harmonic components:

$$
W_{\text {ref }}=W_{N_{\max }}
$$

Then, the acoustic power convergence is studied with respect to the number of retained harmonic component by introducing the following error:

$$
\epsilon_{\mathrm{p}, N}=\left|\frac{W_{N}-W_{\text {ref }}}{W_{\text {ref }}}\right| \text { with } N=1 \ldots N_{\max }
$$

The optimized surface acoustic power $W_{\text {opt }}$ is calculated with the optimized Fourier basis:

$$
W_{\mathrm{opt}}=\sum_{i \in \mathbb{P}} W_{S}\left(\omega_{i}\right)
$$

The relevance of this basis in the acoustic problem is evaluated with the error between $W_{\text {opt }}$ and $W_{\text {ref: }}$ :

$$
\epsilon_{\mathrm{p}, \mathrm{opt}}=\left|\frac{W_{\mathrm{opt}}-W_{\mathrm{ref}}}{W_{\mathrm{ref}}}\right|
$$

\subsection{Application to the single and multi-instability cases}

In this section, the calculation of the optimal Fourier basis is conducted for the cases 1 and 2 .

The dynamic response of the case 1 is composed of the fundamental frequency $f_{1}$ and several harmonic components $2 f_{1}, 3 f_{1}, 4 f_{1}, 5 f_{1}, 6 f_{1}$ and $10 f_{1}$. By using the method detailed in Figure 4 (a), the mean square velocity convergence can be analyzed as shown in Figure 5 . The higher gaps are detected for the zero order and for $f_{1}$. By selecting these components, it is observed that the optimized mean square velocity error $\epsilon_{\mathrm{k}, \mathrm{opt}}$ (i.e. the relative error between the reference and the mean square velocity calculated with the optimized Fourier basis) reaches $0.48 \%$ as illustrated in Figure 5 (a). In order to try to quantify the relevance of the optimized Fourier basis 


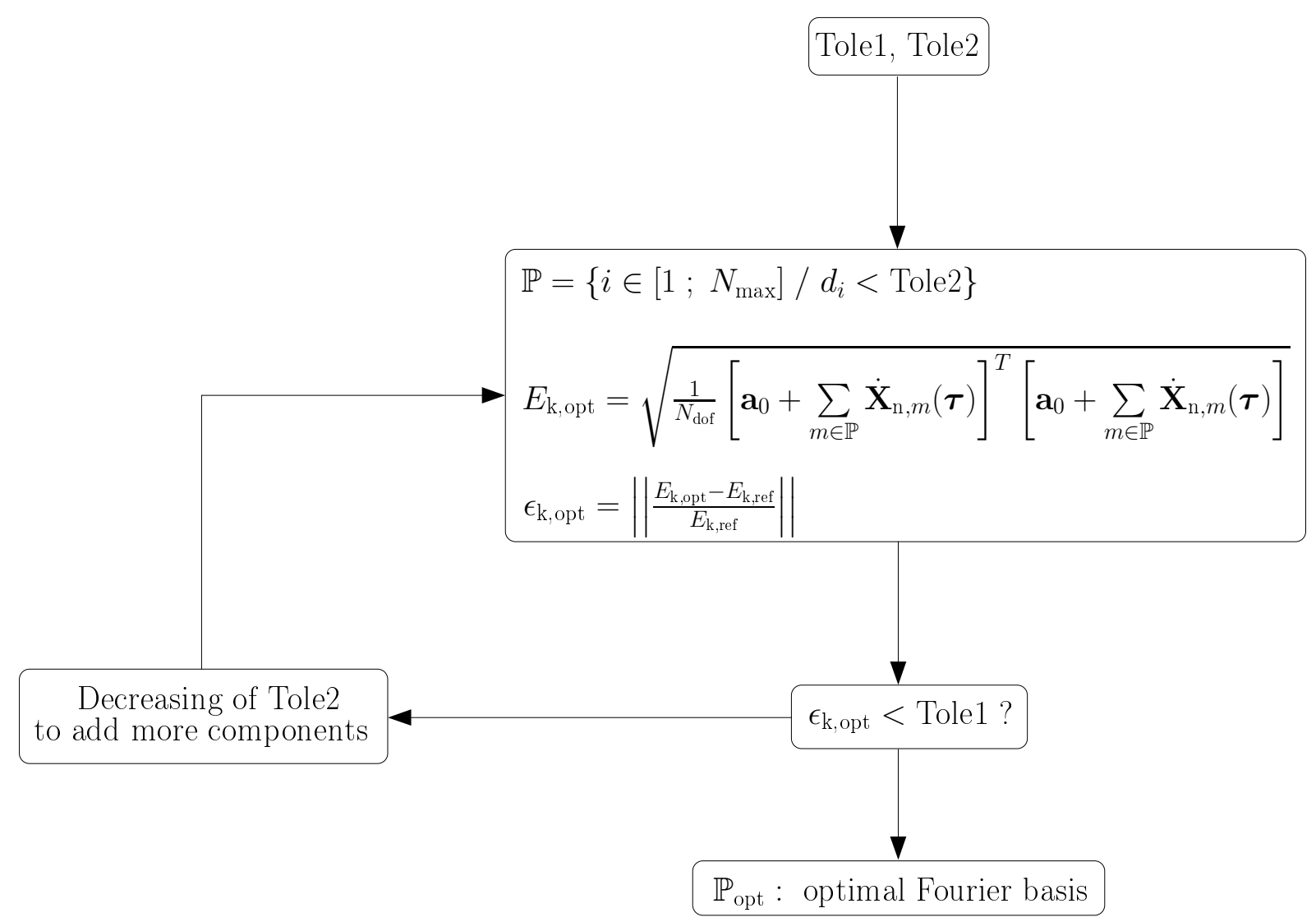

Figure 4: Optimized Fourier basis calculation algorithm

over the acoustic response, the surface acoustic power error $\epsilon_{\mathrm{p}, \mathrm{opt}}$ is calculated with the same components. It is observed in the Figure 5 (a) that the acoustic power is well estimated with only few harmonic components with an error of $0.1 \%$. Adding the less predominant harmonic component $2 f_{1}$ provides a final error $\epsilon_{\mathrm{k} \text {,opt }}$ of $2 \times 10^{-2} \%$ as indicated in Figure 5 (a). Moreover, the acoustic power error reaches $2 \times 10^{-4 \%}$. So, the convergence of the optimized mean square velocity and acoustic power are in good agreement.

The focus is now on the optimal Fourier basis associated with the case 2 during the stationary regime. As previously explained, the Fourier basis of this case is more complex than for the single instability case. Figure 5 (b) shows the mean square velocity and the acoustic power errors for the optimal Fourier basis. For this case, the optimized mean square velocity convergence is slower due to the complexity of the spectrum. However, the iterative process allows to reach a tolerance of about $0.5 \%$ for $\epsilon_{\mathrm{k}, \text { opt }}$ with only five harmonic components. The optimized Fourier basis is also suitable for the acoustic power with a final error of about $10^{-3} \%$.

The optimal Fourier basis associated with the cases 1 and 2, calculated with a tolerance of $5 \times 10^{-2 \%}$ and $0.5 \%$ respectively, is listed in Tables 2 and 3 .

\section{Acoustic approximation method}

The second level of approximation involves the radiations in free space which are calculated with the BEM for each frequency and each field plane. To avoid these calculations, an analytical expression of 3-D directivity of the radiating structure is first determined and several indicators 

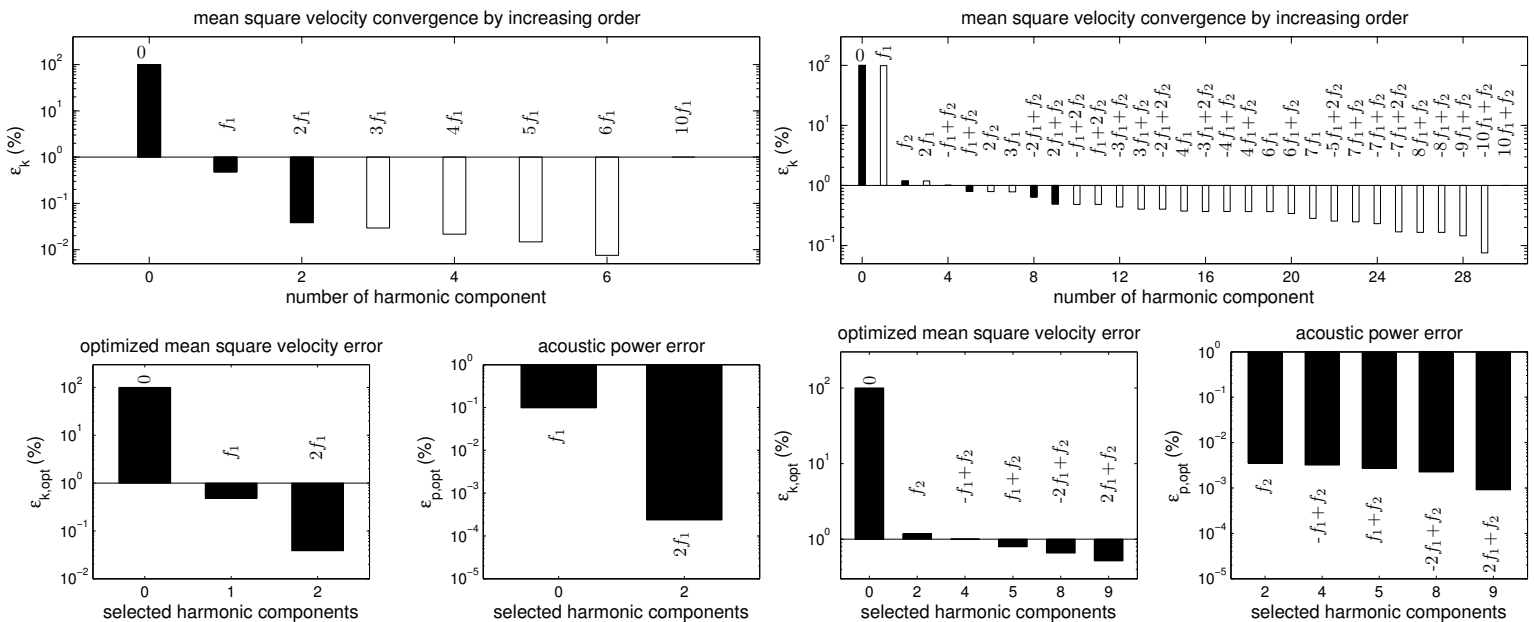

(a) case 1

(b) case 2

Figure 5: Iterative building of the optimized Fourier basis for the cases 1 and 2; black bar: selected harmonic component; white bar: non selected harmonic component. (a): Case 1; (b): case 2 .

\begin{tabular}{ccc}
\hline order & $f_{1}$ & $f_{2}$ \\
\hline 0 & 0 & 0 \\
1 & 1 & 0 \\
2 & 2 & 0 \\
\hline
\end{tabular}

Table 2: Optimal Fourier basis for the case 1 with Tole $1=5 \times 10^{-2} \%$

are introduced to test its validity. Then, the acoustic power levels can be approximated with an appropriated propagation model for every field planes without the BEM.

\subsection{Calculation of the directivity}

As previously explained, the first step of this approach is to well approximate the directivity. So, the focus is on the approximation of the directivity over a sphere which contains the radiating source. As seen in Figure 6 (a), the radiating source $\Omega$ is inside a sphere $S_{R}$ of radius $R$. The sound pressure over $S_{R}$ is denoted by $P(\theta, \phi, \omega)$, where $\theta, \phi$ and $R$ are the spherical coordinates of a point $M$ over the sphere, and $\omega$ denotes the wave frequency. The acoustic intensity $I$ is defined by:

$$
I(\theta, \phi, \omega)=\frac{P(\theta, \phi, \omega) P^{*}(\theta, \phi, \omega)}{\rho c}
$$

where $\rho$ is the density of the air, $c$ denotes the speed of sound in dry air and the star corresponds to the conjugate. The acoustic intensity along a given direction $I_{\text {axe }}$ is also introduced:

$$
I_{\text {axe }}=I\left(\theta_{0}, \phi_{0}, \omega\right)
$$

where $\left(\theta_{0}, \phi_{0}\right)$ denotes a given direction which will be used as a reference and it corresponds to the direction along which the acoustic intensity $I$ is maximum. Finally, the directivity $h$ is 


\begin{tabular}{crc}
\hline order & $f_{1}$ & $f_{2}$ \\
\hline 0 & 0 & 0 \\
\hline 1 & 0 & 1 \\
\hline \multirow{2}{*}{2} & -1 & 1 \\
& 1 & 1 \\
\hline \multirow{2}{*}{3} & -2 & 1 \\
& 2 & 1 \\
\hline
\end{tabular}

Table 3: Optimal Fourier basis for the case 2 with Tole $1=0.5 \%$

calculated by:

$$
h(\theta, \phi, \omega)=\frac{I(\theta, \phi, \omega)}{I_{\text {axe }}}
$$

The function $h$ provides the directions along which the source can radiate. In order to evaluate this function, the Boundary Element Method is used. The sound pressure $P\left(\theta_{i}, \phi_{i}, \omega\right)$ is calculated for all the points $\left(\theta_{i}, \phi_{i}\right) \in S_{R}$ and the discrete directivity $h_{\mathrm{bem}}\left(\theta_{i}, \phi_{i}, \omega\right)$ is calculated.

\subsection{Directivity polynomial fitting}

The main point of this approach is to calculate an analytical expression of the directivity which can be evaluated for all points in free space. The previous BEM calculation only provides the directivity values for a finite number of points over the sphere $S_{R}$. A simple way to approximate this analytical function from its discrete values is to use a polynomial interpolation. The interpolation problem is to determine the optimal degree which provides the best analytical directivity function $h$ :

$$
h(\theta, \phi, \omega)=\sum_{i=0}^{d} \sum_{k=0}^{i} \alpha_{i k} \theta^{k} \phi^{i-k}, \text { with }(\theta, \phi) \in \mathbb{R}
$$

where $\alpha_{i k}$ denotes the unknown coefficients of the $d$-order polynomial. In such a problem, the use of a high polynomial degree provides a well interpolated function over the calculation points but in some case, it generates high oscillations between these points: this is the well known Runge phenomenon. To avoid this phenomenon, the idea is to apply the polynomial interpolation over a part of the known values and then to evaluate the analytical function over all the points. This allows us to determine the best degree in which both minimize the interpolation error and avoid the Runge phenomenon.

The directivity interpolation is performed over $80 \%$ of all the points divided in a random set $M_{\text {rand }}$ and a set composed of the directivity maximum points $M_{\max }$ (Figure $6(\mathrm{~b})$ ). Then, the interpolated function $h$ is evaluated over the whole set composed of the non selected points $M_{\mathrm{C}}, M_{\mathrm{rand}}$ and $M_{\max }$. The optimal polynomial degree is then determined by minimizing the error between the known values and the interpolated function. This problem takes the following form:

$$
\left\{\begin{array}{l}
\text { Find } d \in \mathbb{N} \text { minimizing } \\
\left\|h(M, \omega)-h_{\mathrm{bem}}(M)\right\|_{\infty}=\max _{\left(\theta_{i}, \phi_{i}\right) \in S_{R}}\left|\sum_{i=0}^{d} \sum_{k=0}^{i} \alpha_{i k} \theta_{i}^{k} \phi_{i}^{i-k}-h_{\mathrm{bem}}\left(\theta_{i}, \phi_{i}, \omega\right)\right| \\
\text { with } M=\left(\theta_{i}, \phi_{i}\right) \in S_{R}
\end{array}\right.
$$




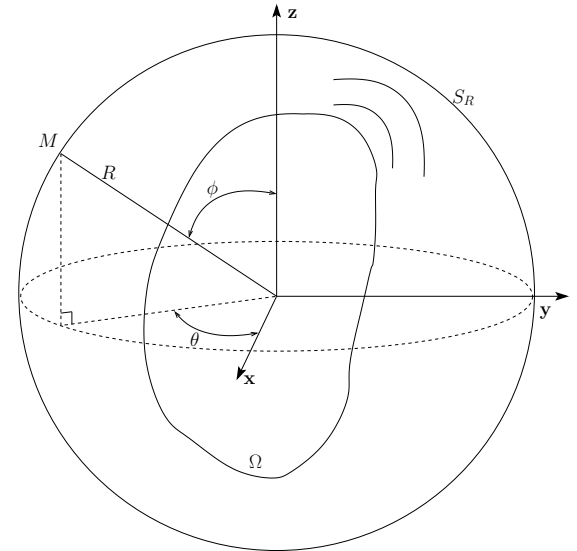

(a)

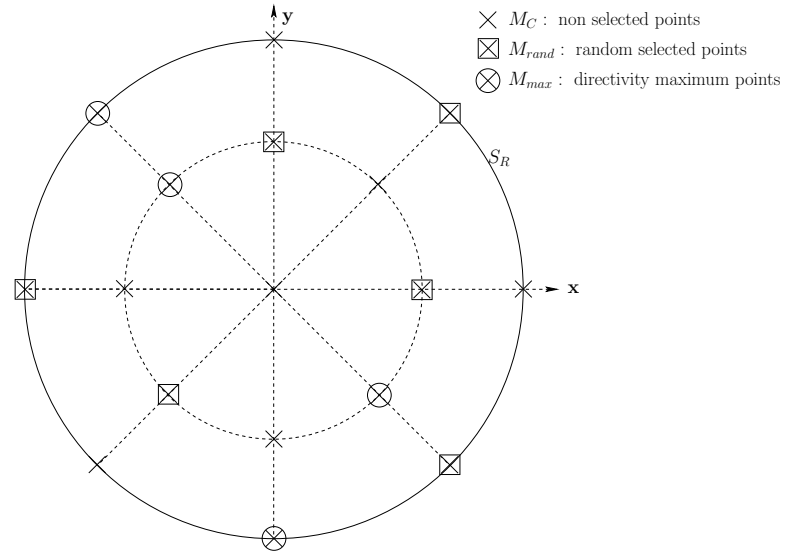

(b)

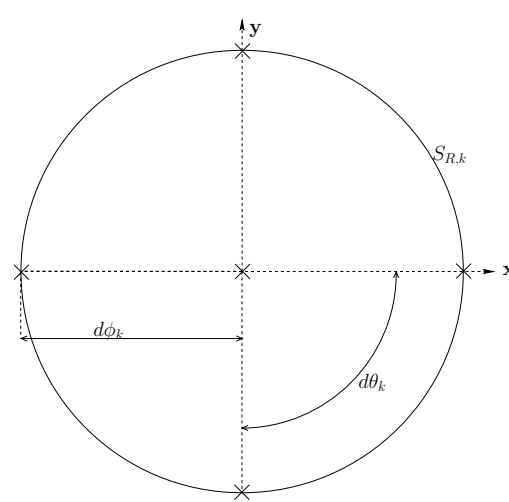

(c)

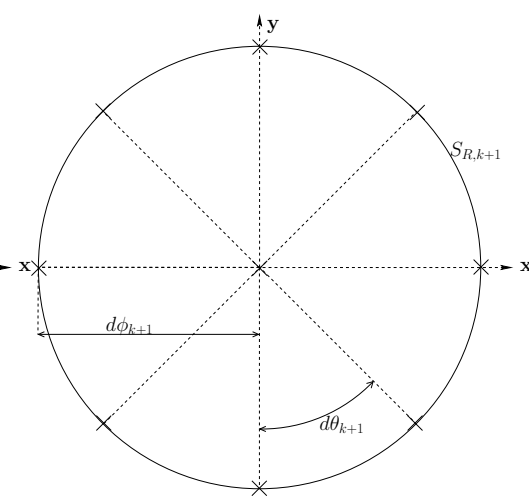

(d)

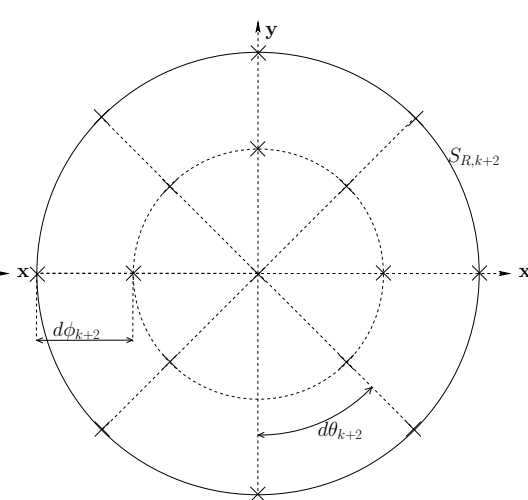

(e)

Figure 6: (a): Draft of the radiating source $\Omega$ in the sphere $S_{R}$; (b): illustration of the selected points for the polynomial interpolation; (c)-(e): sphere refinement method; (c): $S_{R, k}, d \theta_{k}, d \phi_{k}$; (d): $S_{R, k+1}, d \theta_{k+1}, d \phi_{k+1} ;(\mathrm{e}): S_{R, k+2}, d \theta_{k+2}, d \phi_{k+2}$.

However, it is important to note that the interpolation strongly depends on the directivity shape. In this paper, a preliminary work allows to use the polynomial fit. In the general case, preliminary BEM calculations have to be performed to identify the global features of the radiations. However, the previous method can still be applied by changing the interpolation type.

\subsection{Directivity convergence criteria}

The previous method explains how to evaluate the polynomial interpolation quality but does not provide information about the accuracy of the estimated directivity. By refining the sphere $S_{R}$ (i.e. by adding more BEM calculation points), it is possible to improve the interpolated directivity $h$. The issue is now to introduce a criterion to determine the optimal number of BEM calculation points to reach the best directivity. In this section, the focus is on the directivity convergence with respect to the number of BEM calculation points over $S_{R}$.

\subsubsection{Sphere refinement method}

The first step consists of choosing an initial number of BEM calculation points. That is to say, to fix the refinement of the first sphere denoted by $S_{R, 1}$ which contains a "small" number of 
points. For this first sphere, the directivity is calculated by using the interpolation processing method previously defined. Then, the sphere is refined by adding points over $S_{R, 1}$. The $k$-th sphere associated with the refinement $k$ is denoted by $S_{R, k}$ and corresponds to the directivity $h_{k}^{\omega}$. As illustrated in Figures 6 (c),(d) and (e), the successive sphere refinements are of the following form:

- The sphere $S_{R, k}$ is built with the characteristic lengths: $d \theta_{k} ; d \phi_{k}$

- The sphere $S_{R, k+1}$ is built with the characteristic lengths: $d \theta_{k+1}=\frac{d \theta_{k}}{2} ; d \phi_{k+1}=d \phi_{k}$

- The sphere $S_{R, k+2}$ is built with the characteristic lengths: $d \theta_{k+2}=d \theta_{k+1} ; d \phi_{k+2}=\frac{d \phi_{k+1}}{2}$

By successively decreasing the characteristic lengths $d \theta_{k}$ and $d \phi_{k}$, the number of points growth is not too fast and the optimal number of points is accurate. Moreover, it can be noticed that the spheres are imbricated. Thus, the BEM calculations only involved the added points between two refinement steps.

\subsubsection{Global convergence}

The directivity accuracy cannot be estimated over the current sphere because the interpolated directivity minimizes the least square error between the interpolated function and the known values over the sphere. That is why the directivity $h_{k}^{\omega}$ is evaluated over an observation plane $P_{\text {obs }}$ as illustrated in Figure 9. For the $k-1$-th sphere $S_{R, k-1}$, the directivity $h_{k-1}^{\omega}$ is also evaluated over $P_{\text {obs }}$. The global convergence criterion aims at evaluating the error between $h_{k}^{\omega}$ and $h_{k-1}^{\omega}$ over $P_{\text {obs }}$ denoted by $\epsilon_{h, k}^{\omega}$. The sphere refinement is stopped when the previous error reaches the initially fixed tolerance $A_{1}$ :

$$
\left\{\begin{array}{l}
\epsilon_{h, k}^{\omega}=\left\|h_{k}^{\omega}\left(M_{\mathrm{obs}}\right)-h_{k-1}^{\omega}\left(M_{\mathrm{obs}}\right)\right\|_{\infty}<A_{1} \\
\text { with } M_{\mathrm{obs}}=\left(\theta_{\mathrm{obs}}, \phi_{\mathrm{obs}}\right) \in P_{\mathrm{obs}}
\end{array}\right.
$$

\subsubsection{Pattern convergence}

The previous global criterion involves all the point of the observation plane $P_{\text {obs }}$. So, small localized gaps between two successive interpolated directivity are considered and thus this criterion is strict. A less restrictive way to estimate the directivity convergence is to analyze the directivity pattern over the field plane $P_{\text {obs }}$. The directivity $h_{k}^{\omega}$ is evaluated over $P_{\text {obs }}$ and the pattern $\mathscr{C}_{k}^{\omega}$ (which corresponds to the sphere $S_{R, k}$ ) is detected:

$$
\mathscr{C}_{k}^{\omega}\left(M_{\text {obs }}\right)=\left\{\begin{array}{l}
1 \text { if } h_{k}^{\omega}\left(M_{\text {obs }}\right) \geq \bar{h}_{k}^{\omega}\left(M_{\text {obs }}\right) \\
0 \text { otherwise }
\end{array}\right.
$$

where the bar denotes the mean. For the reader comprehension, a draft of the pattern detection is given in Figure 9. The sphere refinement is stopped when the relative error between the patterns $\mathscr{C}_{k}^{\omega}$ and $\mathscr{C}_{k-1}^{\omega}$, denoted by $\epsilon_{\mathrm{C}, k}^{\omega}$, reaches the tolerance $\gamma_{\%}$ as illustrated in Figure 7 (a). Moreover, the gradient of the relative error $\epsilon_{\mathrm{C}, k}^{\omega}$ must be less than the tolerance $\gamma_{\delta}$ to obtained the stabilization of the solution (see Figure $7(\mathrm{~b})$ ). The relative error between two successive patterns takes the following form:

$$
\epsilon_{\mathrm{C}, k}^{\omega}=\frac{\operatorname{card}\left\{\left(\theta_{\mathrm{obs}}, \phi_{\mathrm{obs}}\right) \in P_{\mathrm{obs}} /\left|\mathscr{C}_{k}^{\omega}-\mathscr{C}_{k-1}^{\omega}\right|=1\right\}}{\operatorname{card}\left(P_{\mathrm{obs}}\right)}
$$

where card denotes the number of elements of a given set. $\epsilon_{\mathrm{C}, k}^{\omega}$ corresponds to the percentage of added information between two successive refinements. It does not consider the values of the 


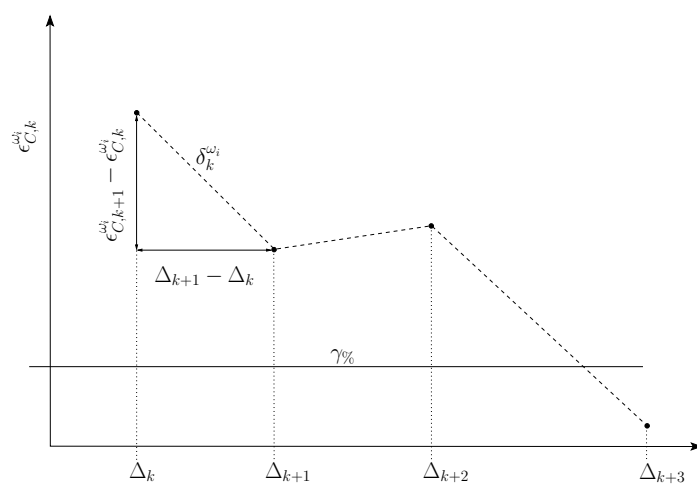

(a)

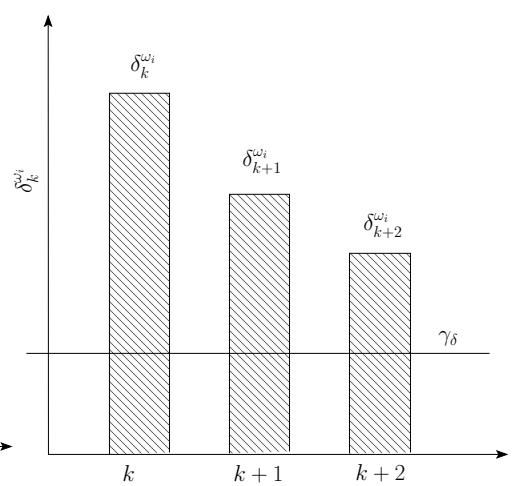

(b)

Figure 7: Illustration of the directivity convergence criterion involving the detected pattern. (a): Relative error between two patterns $\epsilon_{C, k}^{\omega}$ with respect to the number of added points $\Delta k$; (b): draft of the error gradient $\delta_{k}^{\omega}$

directivity but only its shape over a given plane: that is why it is less restrictive than the first criterion. Therefore, the directivity convergence criterion has the following expression:

$$
\epsilon_{\mathrm{C}, k}^{\omega} \leq \gamma_{\%} \text { and } \delta_{k}^{\omega} \leq \gamma_{\delta} \text { with } \delta_{k}^{\omega}=\frac{\epsilon_{\mathrm{C}, k+1}^{\omega}-\epsilon_{\mathrm{C}, k}^{\omega}}{\Delta_{k+1}-\Delta_{k}}
$$

where $\gamma_{\%}$ and $\gamma_{\delta}$ denote the tolerance of the relative error and its gradient respectively, $\Delta_{k}$ corresponds to the number of added points between the sphere $S_{R, k+1}$ and $S_{R, k}$ :

$$
\Delta_{k}=\operatorname{card}\left(S_{R, k+1}\right)-\operatorname{card}\left(S_{R, k}\right)
$$

These two criteria will be used to evaluate the directivity convergence for all the detected harmonic components $\omega_{i}$. The comparison between the two convergence results will allow to estimate the sensitivity of the approximated acoustic levels with respect to the convergence criterion. An overview of the directivity calculation is given in Figure 8.

\subsection{Acoustic power level reconstruction}

The Boundary Element Method provides the sound pressure at every point in the free space. So, it is possible to directly calculate the level of acoustic pressure $L_{\mathrm{p}, \mathrm{bem}}^{\omega}$ for a given harmonic component. In this simplified approach, the pressure is not known and the focus is on the acoustic power $W$ and its levels $L_{W}=10 \log _{10}\left(W / W_{\text {ref }}\right)$, where $W_{\text {ref }}=10^{-12}$ watt denotes the reference acoustic power. The aim of this method is to reconstruct the acoustic power and to calculate the levels $L_{W}$ to estimate the noise radiations in free space. The acoustic power of a given harmonic component $\omega$ associated with the directivity $h_{k}^{\omega}$ is denoted by $W_{k}^{\omega}$. The associated levels in decibels corresponds to the variable $L_{W, k}^{\omega}$. Three information are necessary to calculate $W_{k}^{\omega}$ at every points:

- the directivity $h_{k}^{\omega}$ which is calculated with the previously presented polynomial fit

- the input surface acoustic power vector $\boldsymbol{\Phi}^{\omega}$ which corresponds to $\Phi_{i}^{\omega}=\frac{1}{2} \Re\left[P_{S, i} \dot{X}_{\mathrm{n}, i}^{*}\right]$, where $\mathbf{P}_{S}$ and $\dot{\mathbf{X}}_{\mathrm{n}}$ denote the surface pressure and the surface normal velocity vectors respectively 


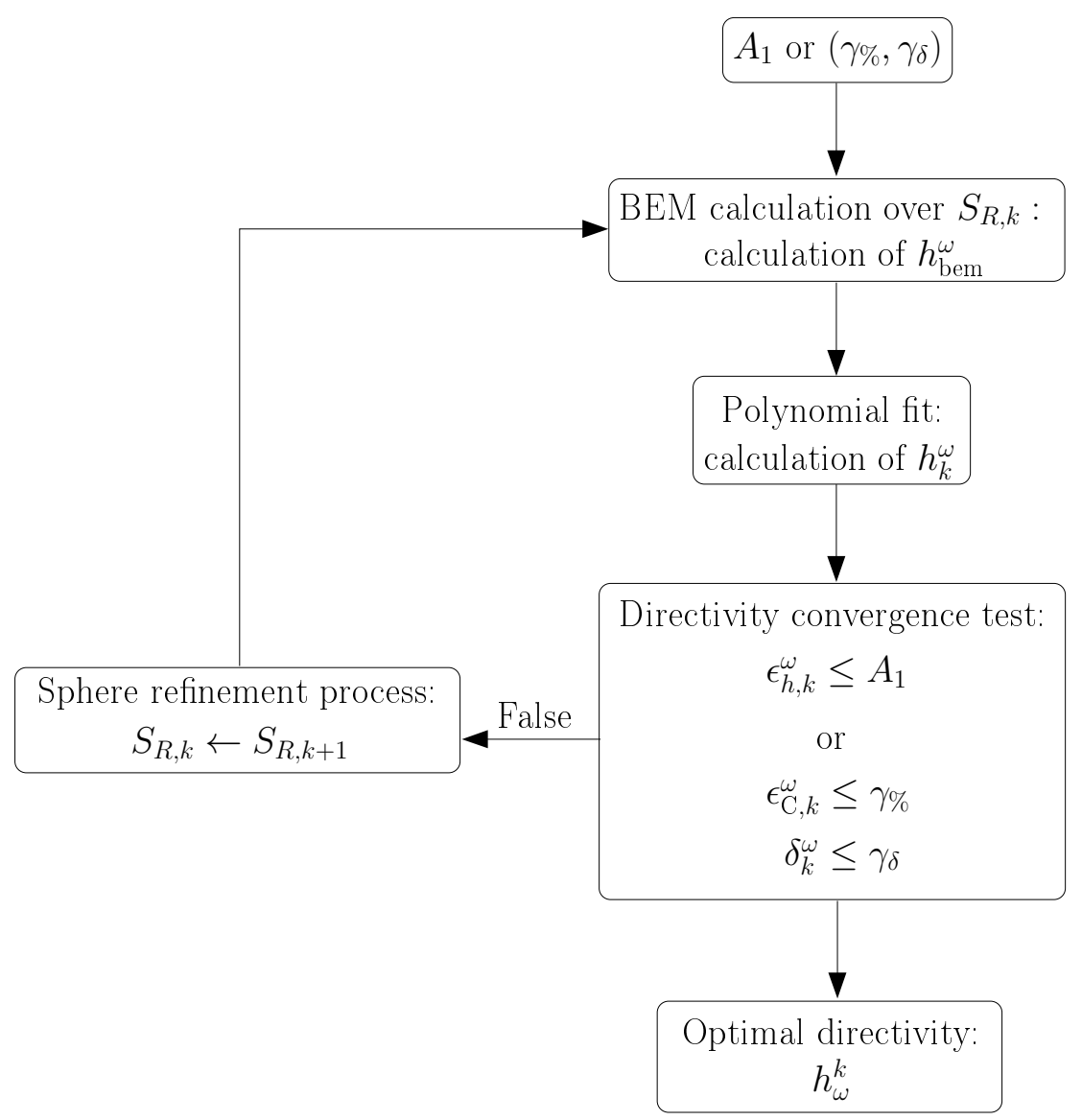

Figure 8: Calculation of the interpolated directivity: polynomial fitting and convergence test

- a propagation model describing the noise level decrease with the distance from the source

Involving the input surface acoustic power, it is directly obtained with the surface pressure calculated by the Boundary Element Method, and the surface normal velocity field calculated by temporal integration. The first approximation used in this approach is to consider that the radiating source $\Omega$ is a punctual source $\Omega_{\mathrm{p}}$. That is to say, the source $\Omega$ generating the acoustic power vector $\boldsymbol{\Phi}^{\omega}$ is replaced with a punctual source $\Omega_{\mathrm{p}}$ with the scalar acoustic power $\Phi_{\mathrm{p}}^{\omega} . \Omega_{\mathrm{p}}$ is located at the mean position of $\Omega$ and $\Phi_{\mathrm{p}}^{\omega}$ corresponds to the average of $\boldsymbol{\Phi}^{\omega}$.

Moreover, a classical propagation model is to consider that the acoustic power in free space $W_{k}^{\omega}$ decreases as $\frac{1}{R_{o b s}^{2}}$ where $R_{\text {obs }}$ is the distance from the source $\Omega_{\mathrm{p}}$. Then, the acoustic power in free space is calculated with the following expression:

$$
W_{k}^{\omega}(M)=\Phi_{\mathrm{p}}^{\omega} S_{\Omega} \frac{h_{k}^{\omega}(M)}{R_{\mathrm{obs}}^{2}}
$$

where $S_{\Omega}$ denotes the surface of the source $\Omega$. The variable $M$ denotes a point in the free space and $R_{\mathrm{obs}}$ is the distance of $M$ from the punctual source $\Omega_{\mathrm{p}}$. Then, the acoustic power levels $L_{W, k}^{\omega}$ are calculated and compared with the sound pressure levels $L_{\mathrm{p}, \text { bem }}^{\omega}$ calculated with the Boundary Element Method. The acoustic power is calculated for each harmonic components and the global acoustic power field $W_{\text {tot }}$ is obtained by superposition. 


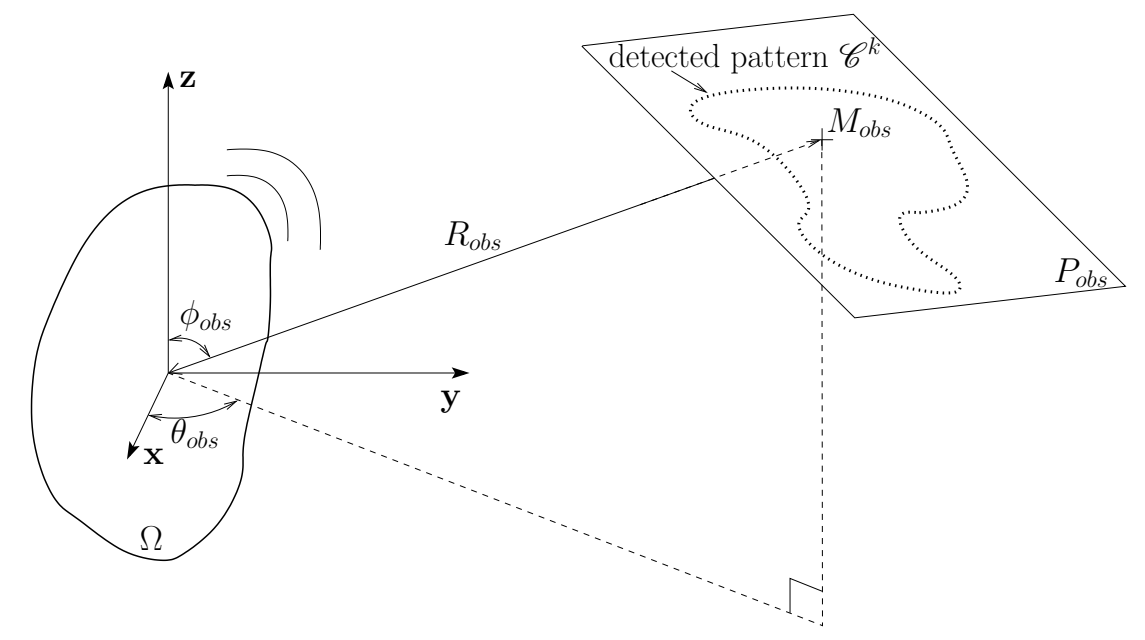

Figure 9: Draft of the parameters used for the directivity convergence criteria

\subsection{Solution accuracy: partial BEM}

The presented method allows us to calculate the acoustic power but does not provide an error estimation of the approximated solution. In this section, a method which allows us to quickly estimate the accuracy of $L_{W, k}^{\omega}$ is presented. The main idea is to use the Boundary Element Method for a "small" set of points. The set of points is determined by detecting the pattern $\mathscr{C}_{W, k}^{\omega}(M)$ of $L_{W, k}(M)^{\omega}$ over the observation plane under study:

$$
\mathscr{C}_{W, k}(M)^{\omega}=\left\{\begin{array}{l}
1 \text { if } L_{W, k}^{\omega}(M) \geq \bar{L}_{W, k}^{\omega}(M) \\
0 \text { otherwise }
\end{array}\right.
$$

Then, the BEM is used only over the boundary of $\mathscr{C}_{W, k}(M)^{\omega}$, denoted by $\partial \mathscr{C}_{k}^{\omega}$, which is composed of a small number of points. Finally, the error estimation consists of comparing $L_{W, k}^{\omega}$ and $L_{\mathrm{p}, \mathrm{bem}}^{\omega}$ over this set of points denoted by $M_{\text {iso }}$.

Another set of points is also used to quantify the quality of the approximated acoustic power. It is composed of all the point inside a circular disc centered over the maximum of $L_{W, k}^{\omega}$ denoted by $M_{\text {disc }}$. This set of points aims at locally evaluating the error near the main areas of propagation.

Three relative errors will be used in the next section:

$$
\begin{aligned}
\epsilon_{\mathrm{bem}}^{\omega} & =\left\|L_{W, k}^{\omega}-L_{\mathrm{p}, \text { bem }}^{\omega}\right\|_{\infty} \\
\epsilon_{\mathrm{disc}}^{\omega} & =\left\|L_{W, k}^{\omega}\left(M_{\text {disc }}\right)-L_{\mathrm{p}, \mathrm{bem}}^{\omega}\left(M_{\mathrm{disc}}\right)\right\|_{\infty} \\
\epsilon_{\text {iso }}^{\omega} & =\left\|L_{W, k}^{\omega}\left(M_{\mathrm{iso}}\right)-L_{\mathrm{p}, \mathrm{bem}}^{\omega}\left(M_{\mathrm{iso}}\right)\right\|_{\infty}
\end{aligned}
$$

where $\epsilon_{\mathrm{bem}}^{\omega}$ denotes the relative error with the complete BEM solution, $\epsilon_{\mathrm{disc}}^{\omega}$ corresponds to the relative error with the partial BEM calculation for the disc and $\epsilon_{\text {iso }}^{\omega}$ is the relative error with the partial BEM calculation for the pattern boundary.

\section{Application to single and multi-instability cases}

In this section, the acoustic approximation method is applied for the optimized basis associated with the cases 1 and 2. For the two cases under study, the directivity interpolation method 


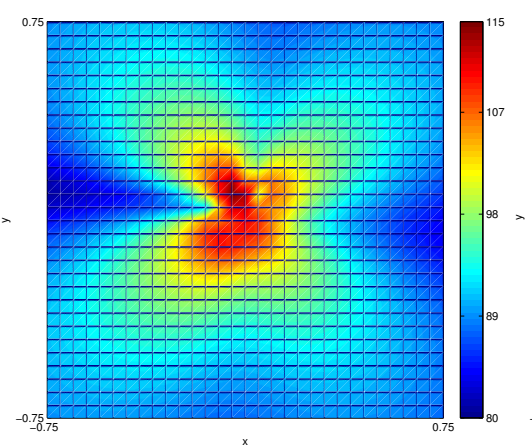

(a)

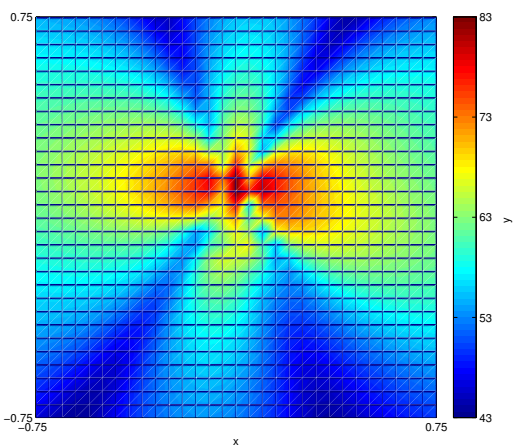

(b)

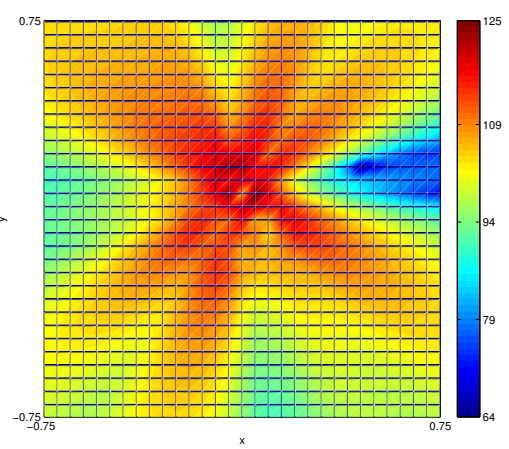

(c)

Figure 10: Sound pressure levels in the free field calculated with the BEM. (a): Case 1- $L_{p, b e m}^{\omega_{1}}$; (b): Case 1- $L_{p, b e m}^{\omega_{2}}$; (c): Case 2- $L_{p, b e m}^{\omega_{4}}$.

presented in Section 4.1 is applied for each harmonic component of the optimized Fourier basis. The directivity convergence is investigated with the criteria defined in Section 4.3. Then, the acoustic power levels are calculated with Equation 32 for a field plane of $1.5 \times 1.5 \mathrm{~m}$, located at $5 \times 10^{-2} \mathrm{~m}$ centered over the disc and in the $(\mathbf{x}, \mathbf{y})$ plane. The reconstructed acoustic power levels accuracy is estimated with the direct BEM results, and the two partial BEM solutions.

As previously explained, the focus is on the outside noise radiations. Therefore, the area of interest is along the $\mathbf{z}$-direction and this allows us to focus on a half sphere centered over the disc with a radius $R=2 \mathrm{~m}$. The directivity does not depend on the distance from the source so the radius $R$ can be arbitrarily chosen. The only limitation is the fact that the sphere must enclose the source. Moreover, the initial number of points over the sphere is set to 256. By using the sphere refinement method described in Section 4.3.1, several half spheres are generated and their features are listed in Table 4.

\begin{tabular}{lcccccccc}
\hline iteration number $k$ & 1 & 2 & 3 & 4 & 5 & 6 & 7 & 8 \\
sphere name $S_{R, k}$ & $S_{R, 1}$ & $S_{R, 2}$ & $S_{R, 3}$ & $S_{R, 4}$ & $S_{R, 5}$ & $S_{R, 6}$ & $S_{R, 7}$ & $S_{R, 8}$ \\
number of points $\operatorname{card}\left(S_{R, k}\right)$ & 256 & 512 & 1024 & 2048 & 4096 & 8192 & 16384 & 32768 \\
\hline
\end{tabular}

Table 4: Features of the half spheres generated with the sphere refinement process

The method will be applied on the cases 1 and 2 with the associated optimized Fourier basis (see Tables 2 and 3). To validate the results, direct BEM calculations are performed over the field plane and the targets are presented in Figure 10. Figures 10 (a) and (b) corresponds to the sound pressure levels associated with the first and the second harmonic components $L_{\mathrm{p}, \text { bem }}^{\omega_{1}}$ and $L_{\mathrm{p}, \mathrm{bem}}^{\omega_{2}}$ respectively. It is observed the presence of four lobes for the two harmonic components with different patterns and shapes. The sound pressure levels associated with the fourth harmonic components of the case $2 L_{\mathrm{p}, \text { bem }}^{\omega_{4}}$ are presented in Figure 10 (c) . Six main directions of propagation are observed. Therefore, the effectiveness of the method is tested by trying to reconstruct the acoustic power level for a "simple" and "complex" propagation patterns (i.e. cases 1 and 2 respectively). 


\subsection{Single instability case}

The optimized Fourier basis associated with this case is given in Table 2. In this simple case, two harmonic components are sufficient to describe the dynamic response and thus the acoustic approximation method is applied for these two frequencies. The directivity associated with the component $\omega_{1}$, denoted by $h_{\mathrm{bem}, k}^{\omega_{1}}$, is presented in Figure 11 (a). As expected, the directivity shape over the sphere is quiet simple and smooth. The quality of the directivity $h_{\mathrm{bem}, k}^{\omega_{1}}$ seems to be not significantly enhanced by refining the sphere. The directivity interpolation process allows to well estimate the directivity as indicated in Figure 11 (b). It is observed that the process is very efficient to interpolate this simple shape.

The convergence of the directivity (i.e. the criterion which allows to stop the sphere refinement process) is now investigated with the two directivity convergence criteria. For each sphere the interpolated directivity is evaluated over a field plane as illustrated in Figure 12. It can be seen that the pattern seems to be well established for a small number of points over the sphere. Actually, it is possible to stop the refinement process at the fourth iteration (i.e. $k=5$ ) regarding the directivity shape. The first criterion which compares two successive directivities over the field plane is used and indicates that the convergence is fast as illustrated in Figure 12 (c). Actually, the associated error $\epsilon_{h, k}^{\omega_{1}}$ is near $0 \%$ for $k=5$ (i.e. for the sphere $S_{R, 6}$ with 8192 points). The second criteria involves the pattern convergence. Figure 12 (b) shows the successive detected patterns and it can be seen that the convergence is also fast. The four lobes shape and orientation are quickly constant. This can also be seen in Figure 12 (d) where the pattern error $\epsilon_{\mathrm{C}, k}^{\omega_{1}}$ is near $0 \%$ for $k=4$ (i.e. for $S_{R, 5}$ with 4096 points). Moreover, the gradient of the previous error denoted by $\delta_{I}^{\omega_{1}}$ quickly converges: $\delta_{3}^{\omega_{1}}$ is about $10^{-4}$ which indicates that the solution is stabilized. To conclude this analysis, the convergence of the directivity has been illustrated on two criteria and for this case, the pattern convergence seems to be the most efficient.

The previous analysis is now performed for the second harmonic component of the optimized Fourier basis associated with the case 1. The directivity is estimated with the BEM and presents two main directions of propagation as indicated in Figure 13 (a). The interpolation process associated with each sphere shows that this directivity can be well represented with few points and the Figure 13 (b) indicates that the process is efficient.

Involving the directivity convergence, Figure 14 (a) shows that the shape is different from the first harmonic components with two main areas of propagation. The associated global error presents irregular variations as indicated in Figure 14 (c) and this criterion provides an acceptable global error $\epsilon_{h, k}^{\omega_{2}}$ of $2 \%$ for the seventh sphere (i.e $S_{R, 7}$ with 16384 points). Involving the pattern convergence, the shape is well established at the sixth iteration associated with $\mathscr{C}_{6}^{\omega_{2}}$ as indicated in Figure 14 (b). The analysis of the pattern error and its gradient shows that the error evolution is smoothed by this criterion (see Figure 14 (d) top). It can be seen that the pattern error associated with the fifth iteration is less than $2 \%$ and the associated gradient (i.e. $\left.\delta_{5}^{\omega_{2}}\right)$ is about $10^{-4}$. So, the pattern error is still more efficient and provides a faster convergence.

As previously explained, the global error $\epsilon_{h, k}^{\omega}$ is more strict than the pattern error and this explains the fact that the pattern error has more regular variations and a faster convergence. Moreover, it can be seen in Figure 14 (a), for $h_{4}^{\omega_{2}}$, that high localized values are present and disturb the global shape. This is a consequence of the interpolation process: in some cases a minimal number of points over the sphere are needed to observe the start of the directivity convergence. Moreover, the determination of the optimal interpolation degree is iteratively performed and this process can be enhanced to improve the directivity convergence. The global error considers those localized values and thus, its evolution is irregular. On the other hand, the pattern error does not consider this numerical phenomenon and this is why its evolution is 
smooth. Therefore, it can be concluded that the pattern error is more efficient to evaluate the directivity convergence.

Another interesting aspect of this method is the fact that the optimal number of BEM calculation points which allows to reach a given error is automatically obtained. Moreover, this optimal number of points can be different for each harmonic component: some frequencies have a "simple" directivity (i.e. as for $\omega_{1}$ ) and others have a complex one (i.e. as for $\omega_{2}$ ). In general, the higher the frequency is, the more complex the directivity pattern is. Therefore, more BEM calculation points will be needed for the high frequency radiations than for the low. However, to limit the number of calculation points, an interesting way is to link the tolerance with the order of the component in the optimized Fourier basis: as the components are sorted by predominance, the tolerance can be "high" for the first few ones and "low" for the last.

\subsubsection{Acoustic power levels reconstruction in free space}

In order to establish the relevance of the approximated directivity, the focus is now on the acoustic power levels reconstruction. This section aims at calculating the levels of acoustic power for each directivity associated with each sphere and for each frequency. The results will allow us to highlight the effectiveness of the pattern convergence error. Moreover, to quantify the accuracy of the reconstructed field, the results will be compared with a direct BEM calculation and the two partial BEM criteria (i.e. the circular patch and the pattern boundary control points).

As previously explained, the acoustic power levels are calculated with Equation 32 and Figure 15 shows the reconstruction of the two harmonic components of the case 1. Involving the levels $L_{W, k}^{\omega_{1}}$ of the first component $\omega_{1}$, Figure 15 (a) indicates that the convergence seems to be fast as for the directivity convergence (see Figure 12). The four lobes quickly appear and are well established since the fourth iteration. By analyzing the target level map (i.e. the direct BEM calculation in Figure 10 (a)), it can be concluded that the target is reached in terms of levels and pattern. The same analysis can be performed for the second harmonic component $\omega_{2}$ : the convergence is still fast, the shape is quickly well established as indicated in Figure 15 (c). Moreover the target presented in Figure 10 (b) is also reached.

\subsubsection{Estimation of accuracy of the approximated solution}

The solution accuracy is now estimated by performing a comparison with the direct BEM calculation, and the two partial BEM solutions (see Equations 34, 35 and 36). Figure 16 (a) shows the relative error between the reconstructed acoustic power levels and the BEM sound pressure levels denoted by $\epsilon_{\mathrm{bem}}^{\omega}$. It is observed that the error does not exceed $9 \%$ for all the components and that the approximation converges. For the stabilized solution, the error is about $6.5 \%$ for $\omega_{2}$ and $2.2 \%$ for $\omega_{1}$. Moreover, it is observed that the solution is stabilized since $S_{R, 5}$ and $S_{R, 6}$ for $\omega_{1}$ and $\omega_{2}$ respectively. These two spheres correspond to the prediction given by the directivity pattern error whereas the global directivity error provides an overestimation of the number of BEM calculation points. Therefore, it can be concluded that the pattern error and its gradient are a relevant and efficient to well estimate the directivity convergence.

However, the main idea of the proposed method is to avoid the use of the BEM. That is why, the criteria involving the partial BEM indicators have been introduced. Involving the circular patch centered over the maximum acoustic power level, the error $\epsilon_{\text {disc }}^{\omega}$ quickly converges as indicated in Figure 16 (b). However, this error estimator provides an overestimation of the final error. Involving the error given by the partial BEM calculation for the pattern boundary, it is observed the same kind of convergence as for the two previous errors but the final value is 
close to the global BEM calculation. From this point of view, it can be concluded that the "isovalue" error is the most efficient. This better error estimation is due to the fact that both the levels and the iso-value shape are included in this indicator and that is why it is more relevant. For the two harmonic components under study for the case 1, an overview of the associated detected iso-value is presented in Figures 15 (b) and (d). For the reader comprehension, it can be noticed that the number of BEM calculation points represents about $10 \%$ of the field points for the two partial BEM criteria.

\subsection{Multi-instability case}

The global method has been applied on the case 1 and validated for two simple directivity patterns. The focus is now on the cases 2 with two unstable modes. The associated optimized Fourier basis is composed of five harmonic components and their radiations are complex. In order to test the method on the most complex case, the focus is on the fourth harmonic component $\omega_{4}$ associated with the frequency $-2 f_{1}+f_{2}$. The radiations over the field plane present six lobes as indicated in Figure 10 (c).

\subsubsection{Directivity calculation}

The directivity interpolation process is applied and the BEM calculations over the spheres highlight a complex directivity with small localized areas of propagation as illustrated in Figure 17 (a). Despite the complexity of the directivity, the polynomial interpolation process is efficient as indicated in Figure 17 (b). However, more points are needed to well represent the directivity shape than for the case 1 . Therefore, a slower convergence can be expected.

The directivity function is now evaluated over the field plane and two convergence criteria are analyzed. Firstly, a proper directivity shape appear only since the fourth iteration as illustrated in Figure 18 (a). The global directivity error $\epsilon_{h, k}^{\omega_{4}}$ presents irregular variations as for the case 1. Moreover, in this case this indicator cannot be used due to the fact that it does not converge. Involving the detected patterns, it is observed that the shape converges: five directions of propagation clearly appear and the sixth appears at the last iteration (see Figure 18 (b)). The pattern error smoothly decreases and this indicator highlights the convergence as indicated in figure 18 (d). The gradient also decreases and this shows the stabilization of the solution. However, as expected, the convergence is slower than for the case due to the complexity of the directivity.

The same analysis is performed for the other components (i.e. $\omega_{1}=2 \pi f_{2}, \omega_{2}=2 \pi\left(-f_{1}+f_{2}\right)$, $\omega_{3}=2 \pi\left(f_{1}+f_{2}\right)$ and $\left.\omega_{5}=2 \pi\left(2 f_{1}+f_{2}\right)\right)$ and the global error does not allow us to stop the sphere refinement process due to irregular variations (results not presented). However, the pattern error and its gradient provide better indications about the directivity convergence as indicated in Figure 19. The error evolution is more smooth for all the harmonic components. Therefore, it is possible to set the tolerances to reach for the error $\epsilon_{\mathrm{C}, k}^{\omega}$ and its gradient $\delta_{I}^{\omega}$. For this criterion, the convergence is still slow due to the complexity of the directivity.

\subsubsection{Acoustic power levels reconstruction in free space}

The focus is now on the acoustic power levels in free space and as previously, only the results involving the fourth harmonic component $\omega_{4}$ are presented. Figure 20 (a) shows the reconstruction of $L_{W, k}^{\omega_{4}}$ for each iteration. It is observed that the shape presents four lobes for the five first

iterations, then a fifth appears and finally the six expected lobes are present. So, the method is able of reconstructing the target BEM solution in terms of levels and shape (see Figure 10 (c)). 
However, as previously explained, the number of iterations is higher than for the case 1 but the optimal number of calculation points is also well estimated.

The accuracy of the solution for each harmonic component is investigated by using the three criteria involving the complete and the two partial BEM calculations. By using the complete BEM calculation, it is observed that the error is less that $8 \%$ as indicated in Figure 21 (a). Moreover, as expected, the error increases with the frequency. So, it can be concluded that the method is efficient even for such a complex case.

The comparison with the partial BEM over the disc provides an error presenting irregular variations as indicated in Figure 21 (b). However, the error values are in accordance with the complete BEM error. Involving the pattern boundary, it is calculated with the BEM on the field points of the pattern boundary $\partial \mathscr{C}_{k}^{\omega}$ as illustrated in Figure 20 (b). This error has the same features as the error with the disc and provides similar values and evolution. Therefore, in this case, it can be concluded that the two error indicators $\epsilon_{\text {disc }}^{\omega}$ and $\epsilon_{\text {iso }}^{\omega}$ are equivalent and both provide a good estimation of the error with the complete BEM calculation.

\subsection{Effectiveness of the proposed approach}

To conclude on the effectiveness of the proposed approach, it is important to note the following points. Firstly, the field plane used in this study contains about 1000 points. The BEM calculation time is about $1 \mathrm{~s}$ per points, i.e. about $16 \mathrm{~min}$. With the proposed method, the acoustic power level reconstruction is close to be instantaneously achieved in this case. Secondly, even if the number of BEM calculation points can be large to achieve the directivity convergence, this calculation is performed only one time per frequency. Moreover, it has been shown that good results are obtained for tolerances which are not "too" strict (i.e. for a small number of points). Finally, this approach needs more time computation for the directivity calculation, but this field allows us to instantaneously reconstruct the free space noise levels without any limitations on the number of field points.

\section{Conclusion}

This work proposes a simplified approach for the calculation of acoustic emission associated with disc brake squeal events. A simplified disc brake model with nonlinear contact and friction laws is investigated. This simplified brake is able of reproducing two classical cases of instabilities with one and two unstable modes for which the noise radiations have been characterized with the multi-frequency acoustic calculation method.

The selection of the predominant harmonic components in the dynamic response based on the mean square velocity is applied on a single and a multi-instability cases. This criterion allows to significantly reduce the number of harmonic components and guarantee the convergence of the associated surface acoustic power. Only two components are needed to well describe the mean square velocity and the surface acoustic power for the case 1 and only five for the second case.

Therefore, the simplified acoustic method is applied for the optimal Fourier basis of each case. For the first case, the directivity interpolation process allows us to well estimate the directivity over a sphere with a "small" number of points. The directivity convergence criterion involving the pattern is very efficient and allows us to stop the sphere refinement process for the optimal number of point. The propagation model is then applied to reconstruct the acoustic power levels in free space and the results are in good agreement with the direct BEM calculations. To control the final error, BEM calculation points are used: a first set composed of a disc over the field plane and another over the boundary of the detected pattern. It is shown that the second one 
provides a better estimation of the solution accuracy for this case. For the two unstable modes case, the directivity is more complex. However, the method is still efficient but the convergence is slower. The directivity is well estimated and the reconstructed field has a excellent quality. However, for this case, the two error indicators are equivalent.

Finally, the coupling between the harmonic component selection and the approximation of the acoustic power in free field is very efficient. It allows us to well reduce the computation time and guarantees a controlled accuracy of the solution.

\section{Acknowledgments}

The first author gratefully acknowledges the French Education Ministry which supports this research. The authors thank the financial support provided by the French National Research Agency through the framework of its project ANR-12-JS09-0009.

\section{References}

[1] K. Soobbarayen, S. Besset, and J.-J. Sinou. Noise and vibration for a self-excited mechanical system with friction. Applied Acoustics, 74:1191-1204, 2013.

[2] J.-J. Sinou. Transient non-linear dynamic analysis of automotive disc brake squeal - on the need to consider both stability and non-linear analysis. Mechanics Research Communications, 37(1):96-105, 2010.

[3] S. Marburg and B. Nolte. Computational Acoustic of Noise Propagation in Fluids. Springer.

[4] M. Bonnet. Boundary Integral Equation Methods for Solids and Fluids. John Wiley \& Sons Ltd, 1999.

[5] J.-J. Sinou, N. Coudeyras, and S. Nacivet. Study of the nonlinear stationary dynamic of single and multi instabilities for disc brake squeal. International Journal of Vehicle Design, 51(1-2):207-222, 2009.

[6] R. Craig and M. Bampton. Coupling of substructures for dynamic analysis. American Institute of Aeronautics and Astronautics Journal, 7(6):1313-1319, 1968.

[7] H. Ouyang, N. Nack, Y. Yuan, and F. Chen. Numerical analysis of automotive disc brake squeal: a review. International Journal of Vehicule Noise and Vibration, 1(3/4):207-231, 2005 .

[8] N.M. Kinkaid, O.M. O'Reilly, and P. Papadopoulos. Automotive disc brake squeal. Journal of Sound and Vibration, 267(1):105-166, 2003.

[9] F. Massi, L. Baillet, O. Giannini, and A. Sestieri. Brake squeal: Linear and nonlinear numerical approaches. Mechanical Systems and Signal Processing, 21:2374-2393, 2007.

[10] H. Lee and R. Singh. Determination of sound radiation from a simplified disk-brake rotor by a semi-analytical method. Noise Control Engineering, 52(5):225-239, 2004.

[11] S. Oberst, J. C. S. Lai, and S. Marburg. Guidelines for numerical vibration and acoustic analysis of disc brake squeal using simple models of brake systems. Journal of Sound and Vibration, 332(9):2284-2299, 2013. 
[12] U. von Wagner, T. Jearsiripongkul, T. Vomstein, G. Chakraborty, and P. Hagedorn. Brake squeal: Modeling and experiments. VDI-Bericht, 1749:96-105, 2010.

[13] J-J. Sinou, A. Loyer, O. Chiello, G. Mogenier, X. Lorang, F. Cocheteux, and S. Bellaj. A global strategy based on experiments and simulations for squeal prediction on industrial railway brakes. Noise Control Engineering, 332(20):5068-5085, 2013. 


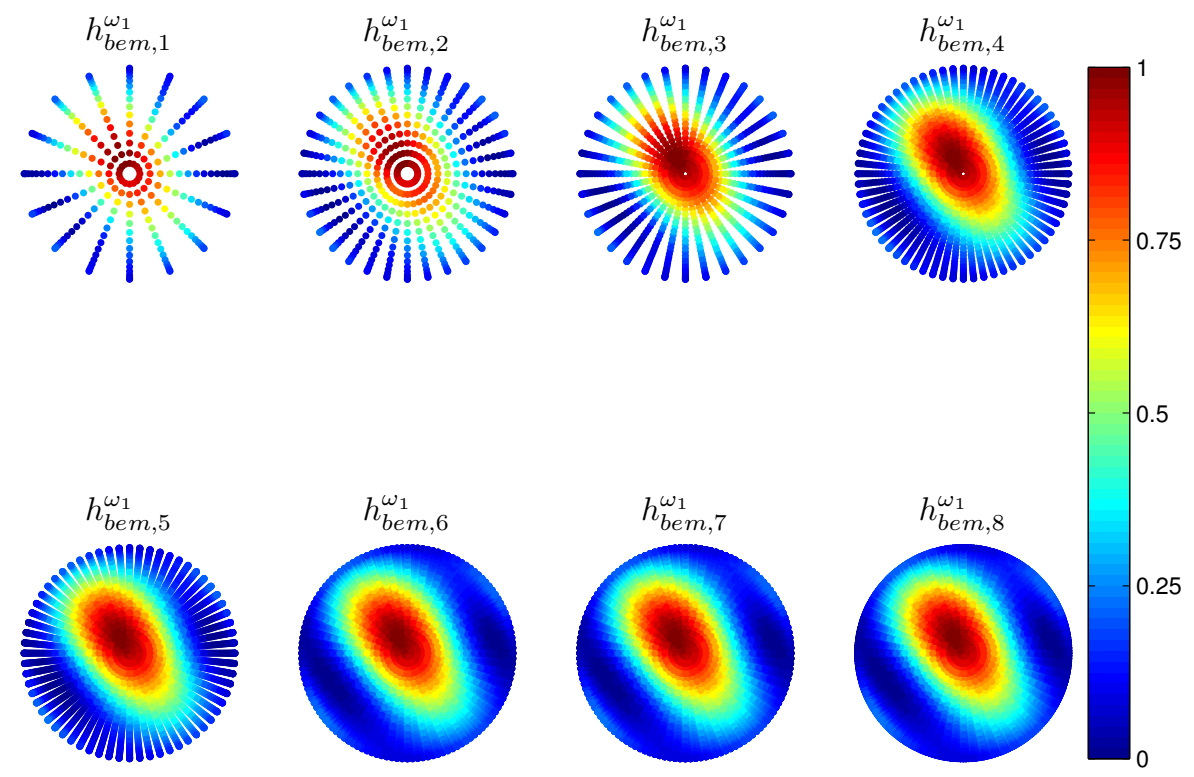

(a)
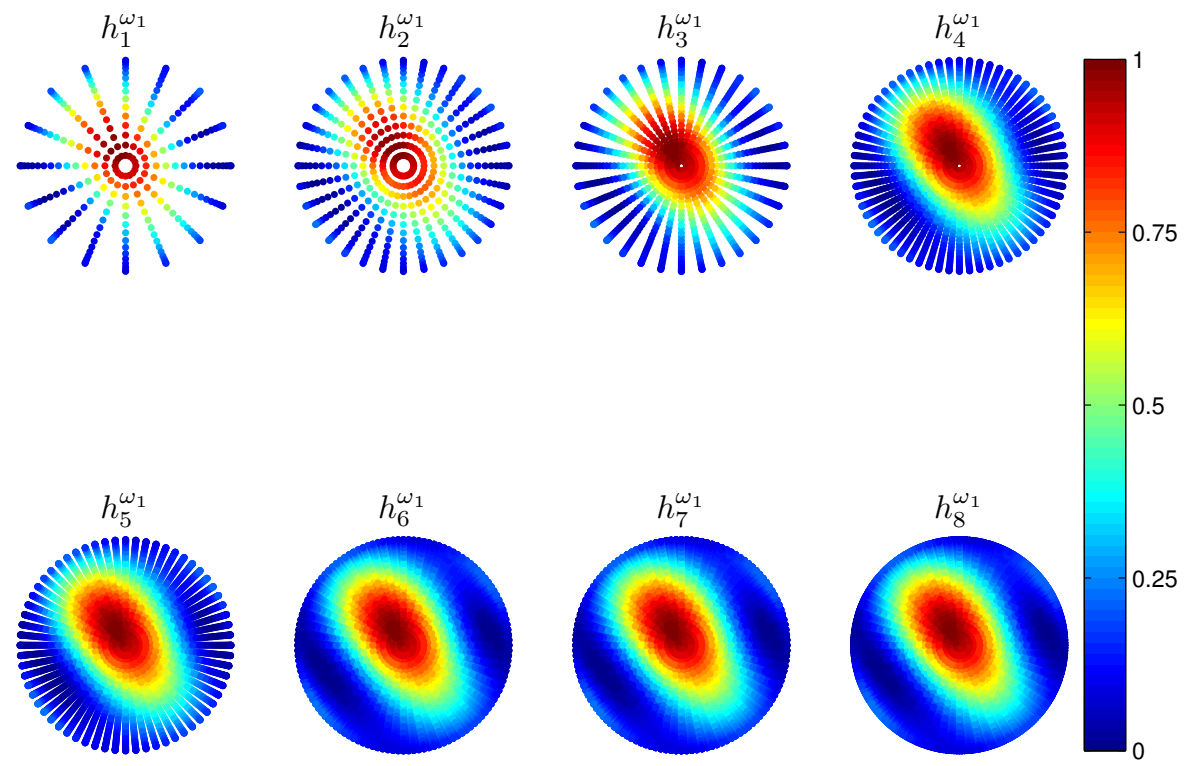

(b)

Figure 11: Directivity polynomial fit over the spheres $S_{R, k}$ for the single instability case: harmonic component $\omega_{1}=2 \pi f_{1}$. (a): Directivity calculated with the BEM $h_{\text {bem }, k}^{\omega_{1}}$; (b): interpolated directivity $h_{k}^{\omega_{1}}$. 

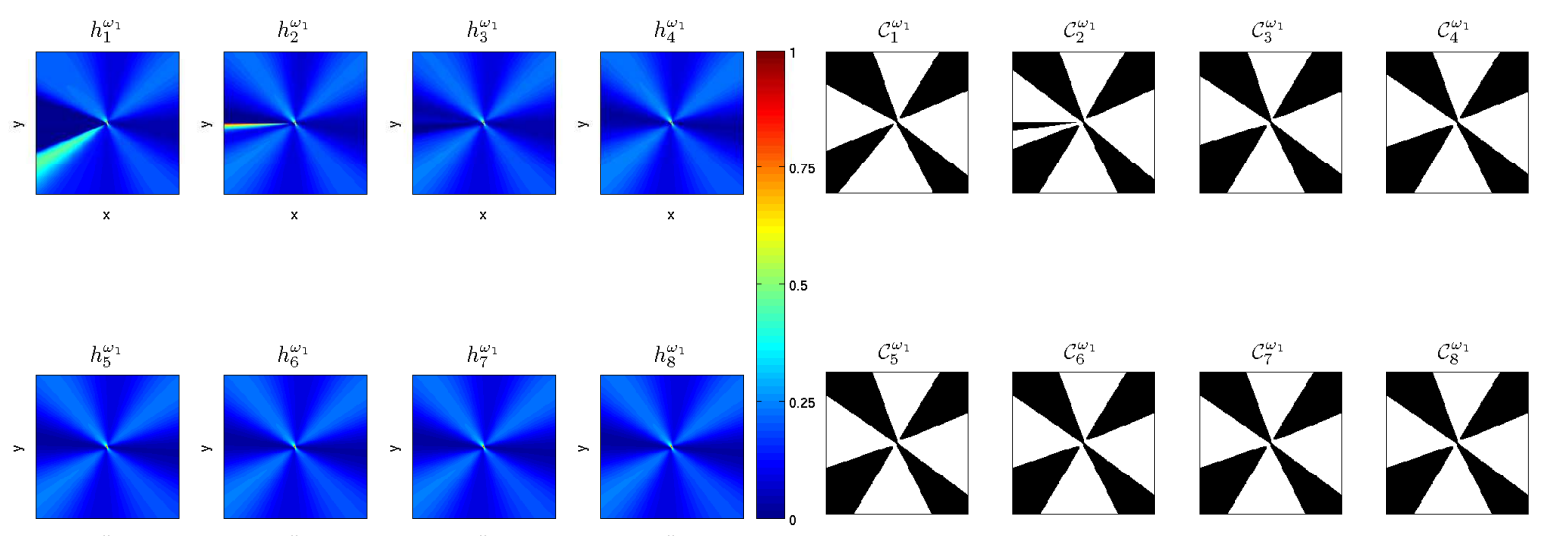

(a)

(b)

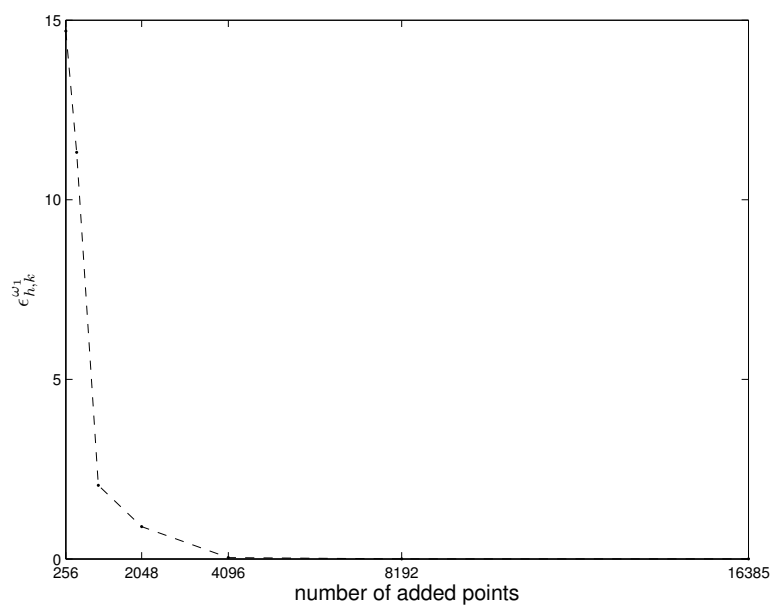

(c)
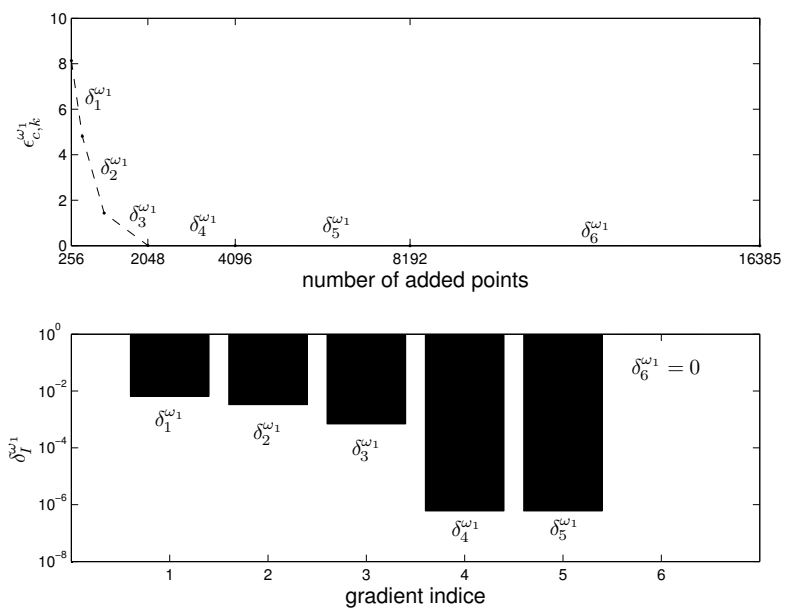

(d)

Figure 12: Analysis of the directivity convergence for the component $\omega_{1}$ of the case 1. (a): Directivity over the field plane; (b): detected pattern; (c): global error $\epsilon_{h, k}^{\omega_{1}} ;(d)$ : top: pattern error $\epsilon_{C, k}^{\omega_{1}}$ - bottom: gradient $\delta_{I}^{\omega_{1}}$. 


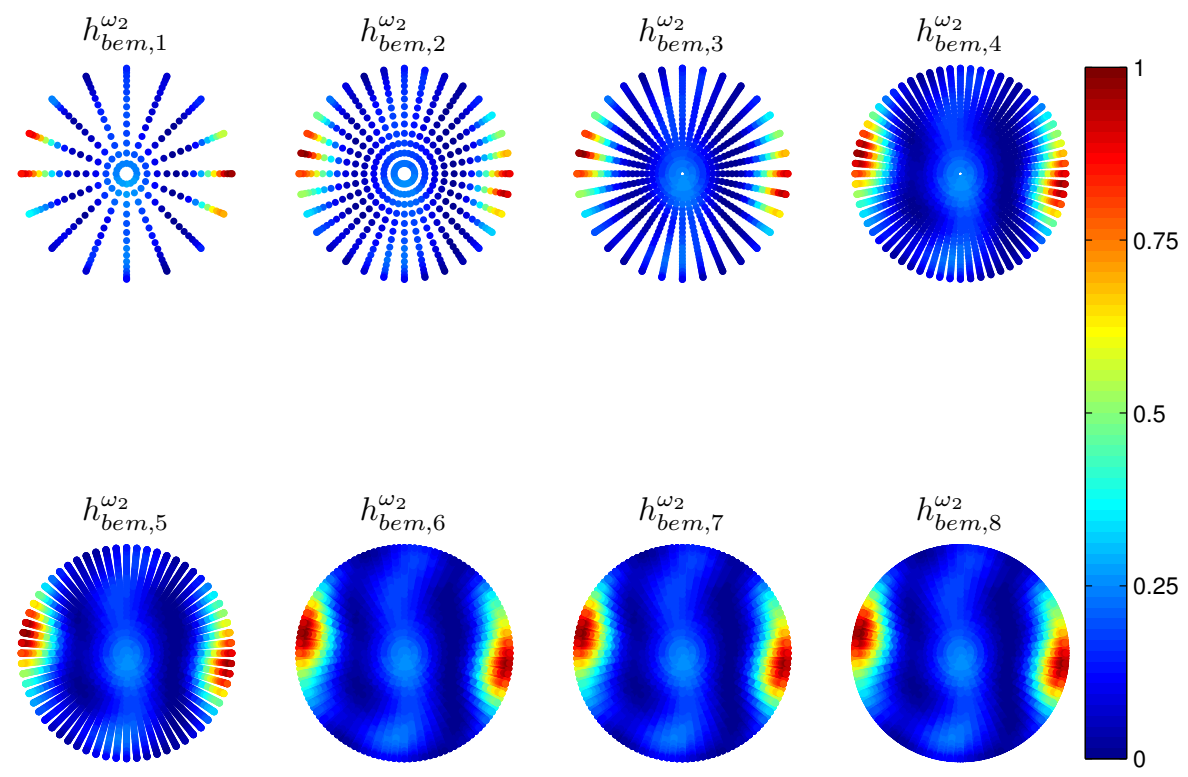

(a)
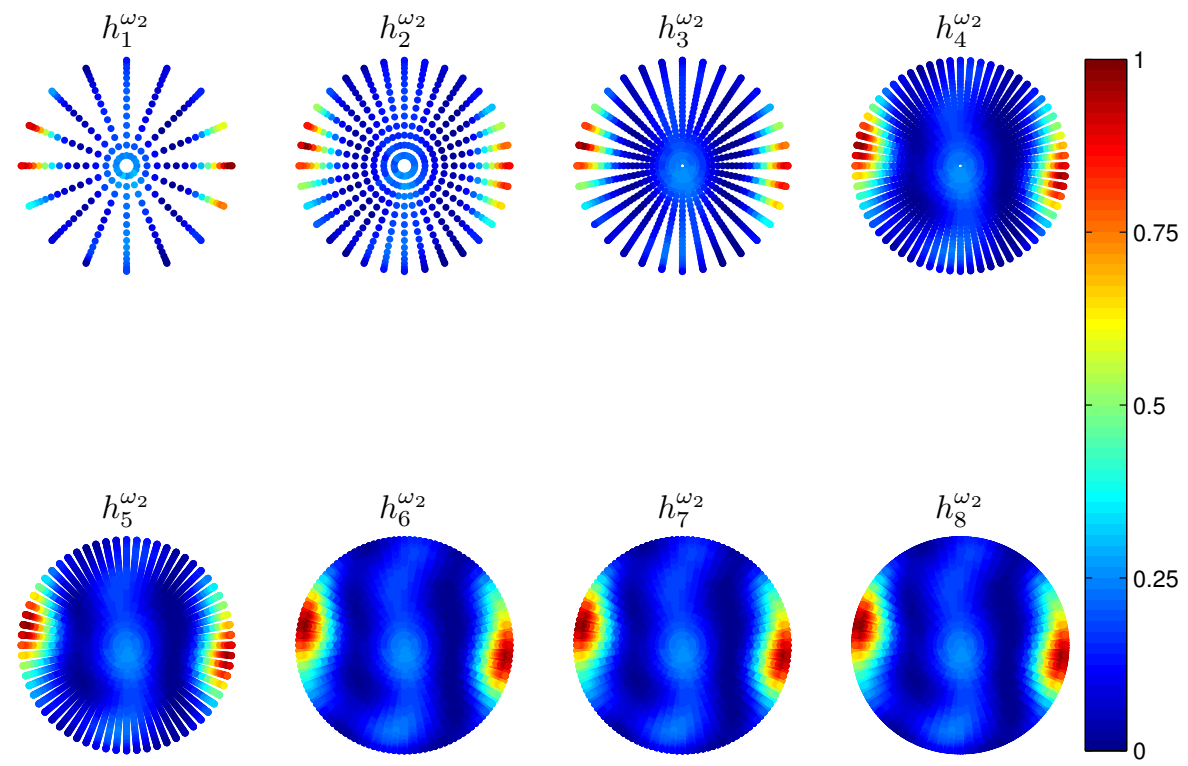

(b)

Figure 13: Directivity polynomial fit over the spheres $S_{R, k}$ for the single instability case: harmonic component $\omega_{2}=4 \pi f_{1}$. (a): Directivity calculated with BEM $h_{\text {bem, },}^{\omega_{2}}$; (b): interpolated directivity $h_{k}^{\omega_{2}}$. 

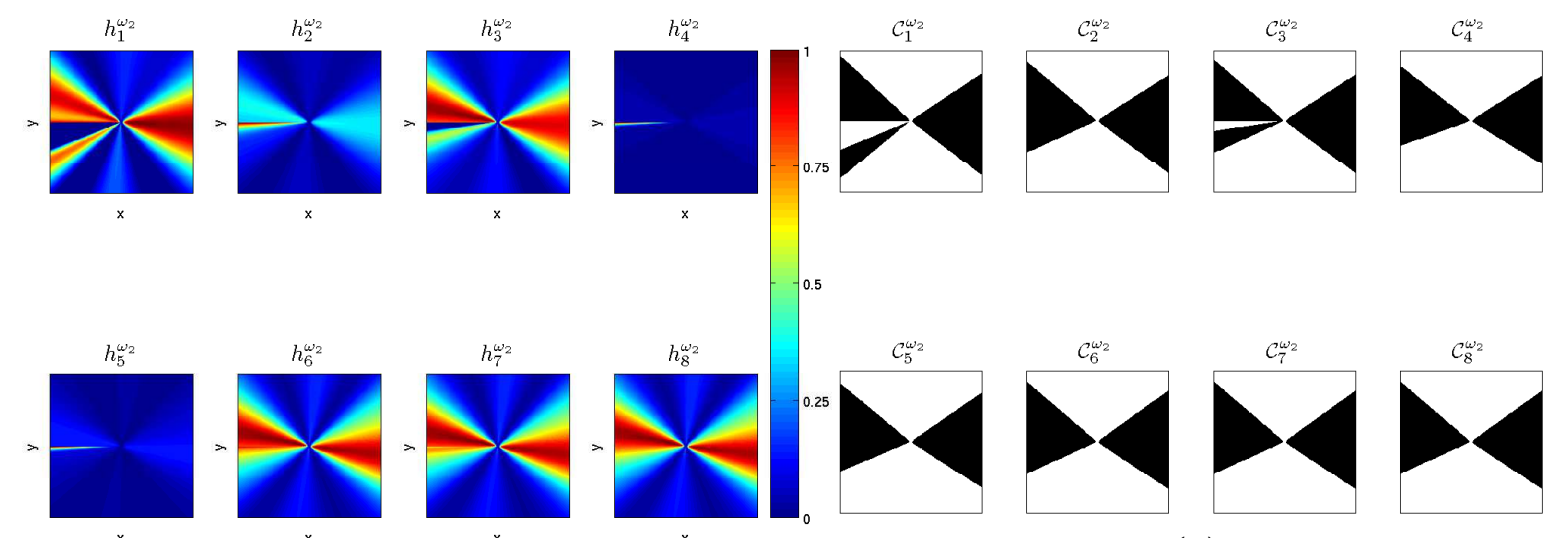

(a) e
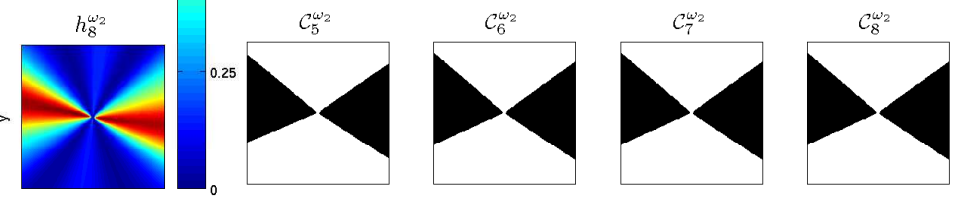

(b)

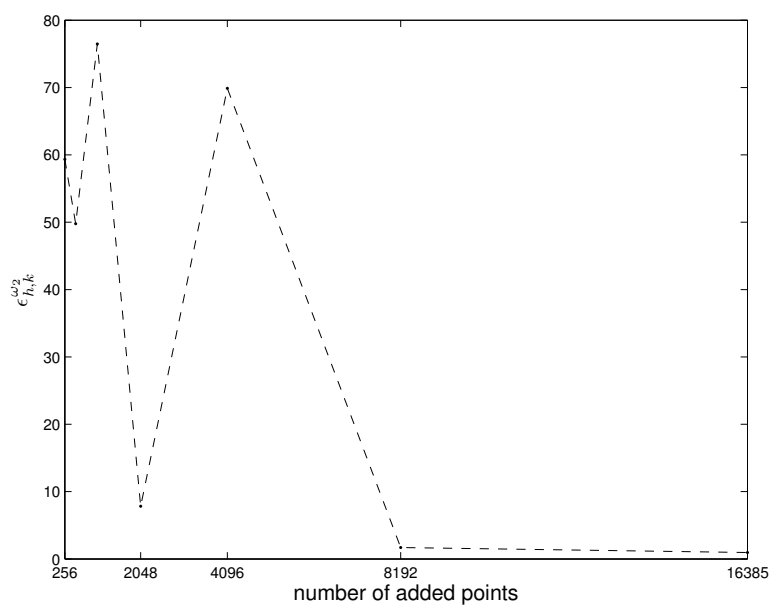

(c)
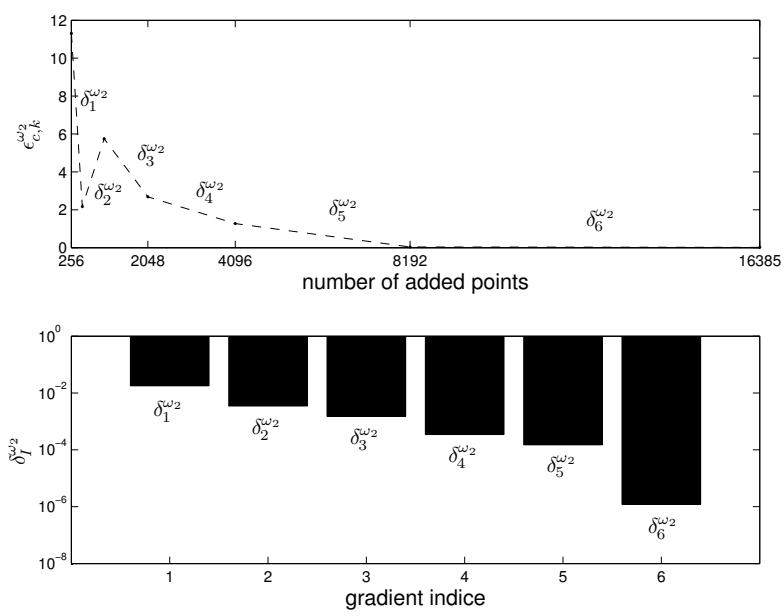

(d)

Figure 14: Analysis of the directivity convergence for the component $\omega_{2}$ of the case 1. (a): Directivity over the field plan; (b): detected pattern; (c): global error $\epsilon_{h, k}^{\omega_{2}}$; (d): top: pattern error $\epsilon_{C, k}^{\omega_{2}}-$ gradient $\delta_{I}^{\omega_{2}}$. 


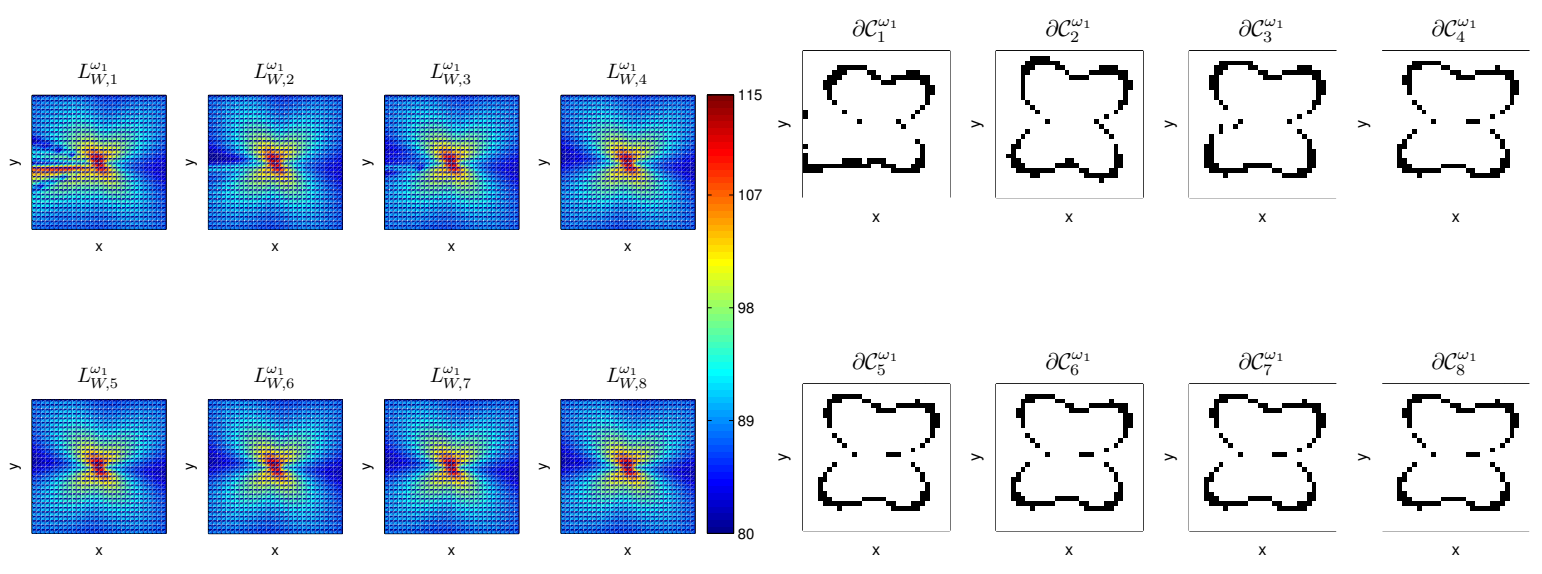

(a)

(b)

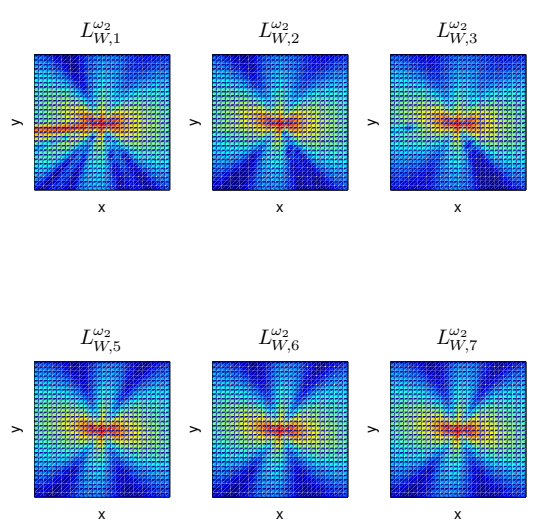

(c)

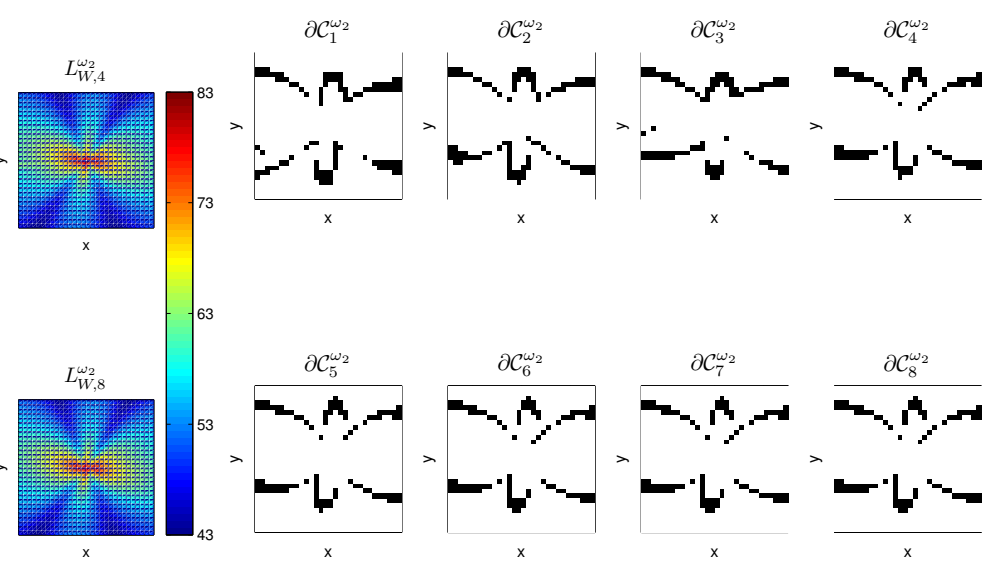

(d)

Figure 15: Acoustic power levels reconstruction for the case 1. (a), (b): Component $\omega_{1}$; (c),(d): component $\omega_{2}$. (a): Acoustic power levels $L_{W, k}^{\omega_{1}}$ : case $1-\omega_{1} ;$ (b): detected iso-value $\partial \mathscr{C}_{k}^{\omega_{1}}$ : case $1-\omega_{1} ;(c)$ : acoustic power levels $L_{W, k}^{\omega_{2}}$ : case $1-\omega_{2} ;(d)$ : detected iso-value $\partial \mathscr{C}_{k}^{\omega_{2}}$ : case $1-\omega_{2}$. 


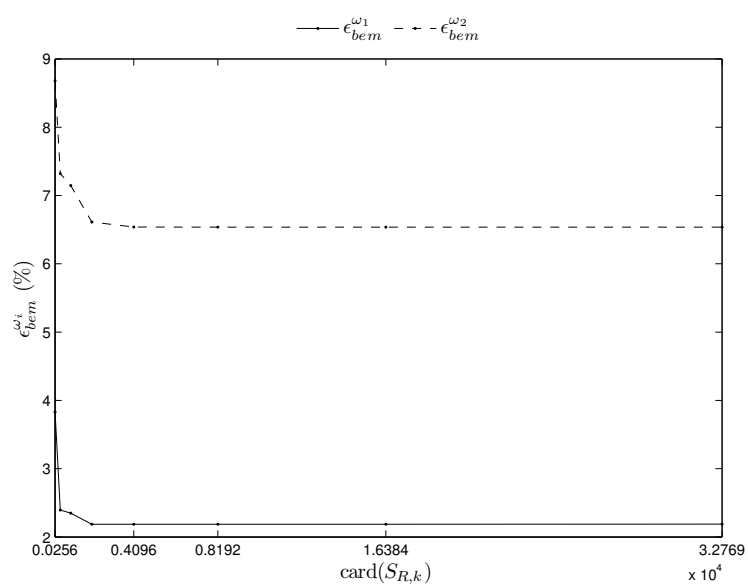

(a)

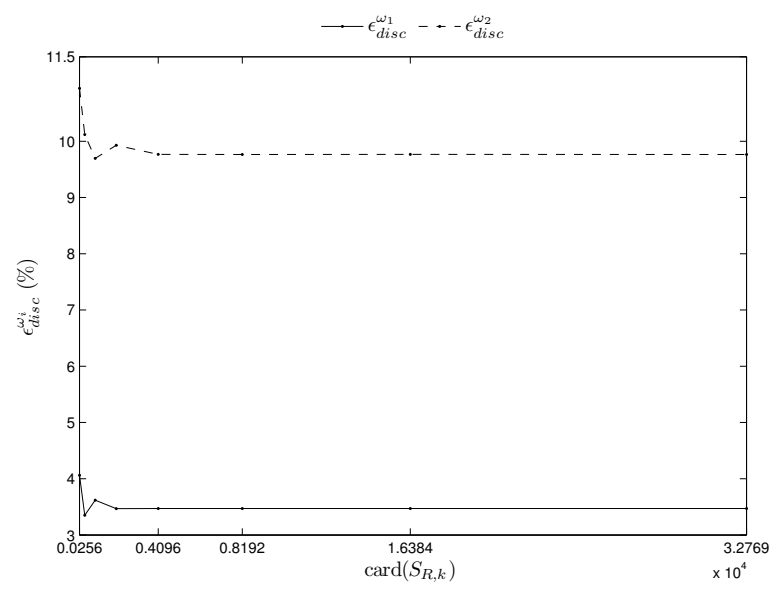

(b)

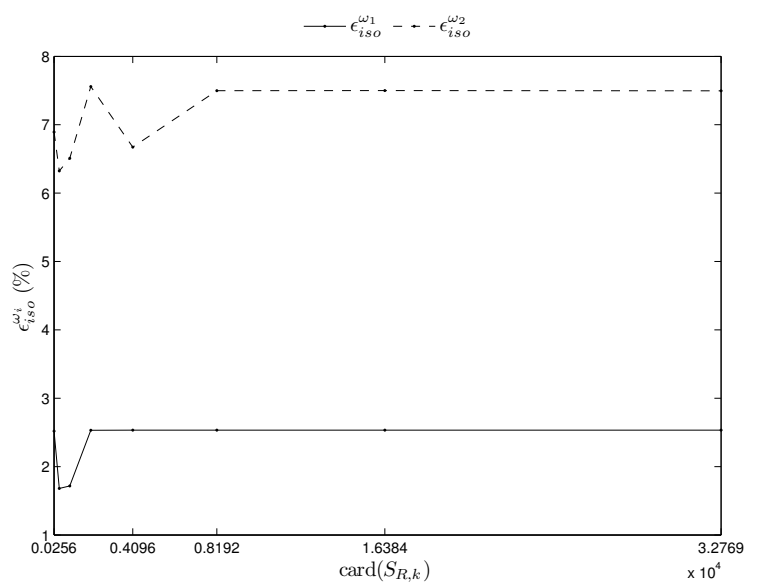

(c)

Figure 16: Accuracy estimation of the approximated acoustic power levels for the case 1. (a): Comparison with the direct BEM calculation; (b): comparison with the partial BEM: disc; (c): comparison with the partial BEM: iso-value. 


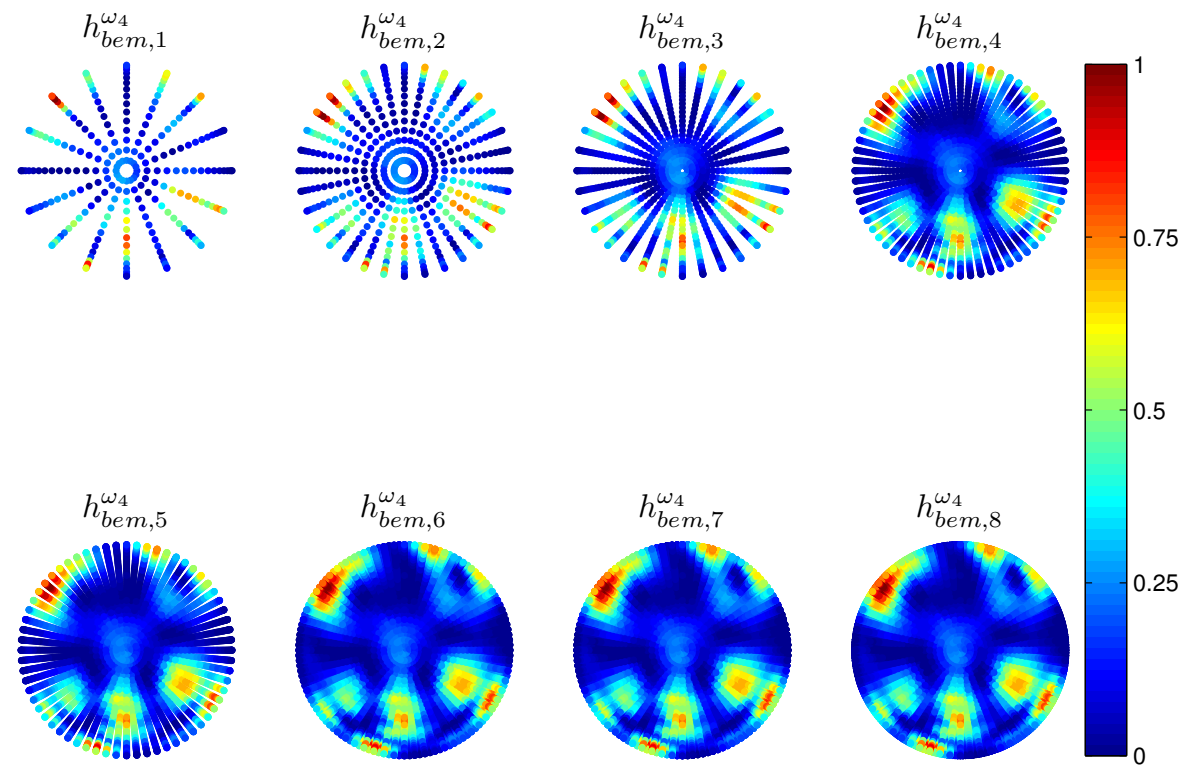

(a) Direct BEM calculation
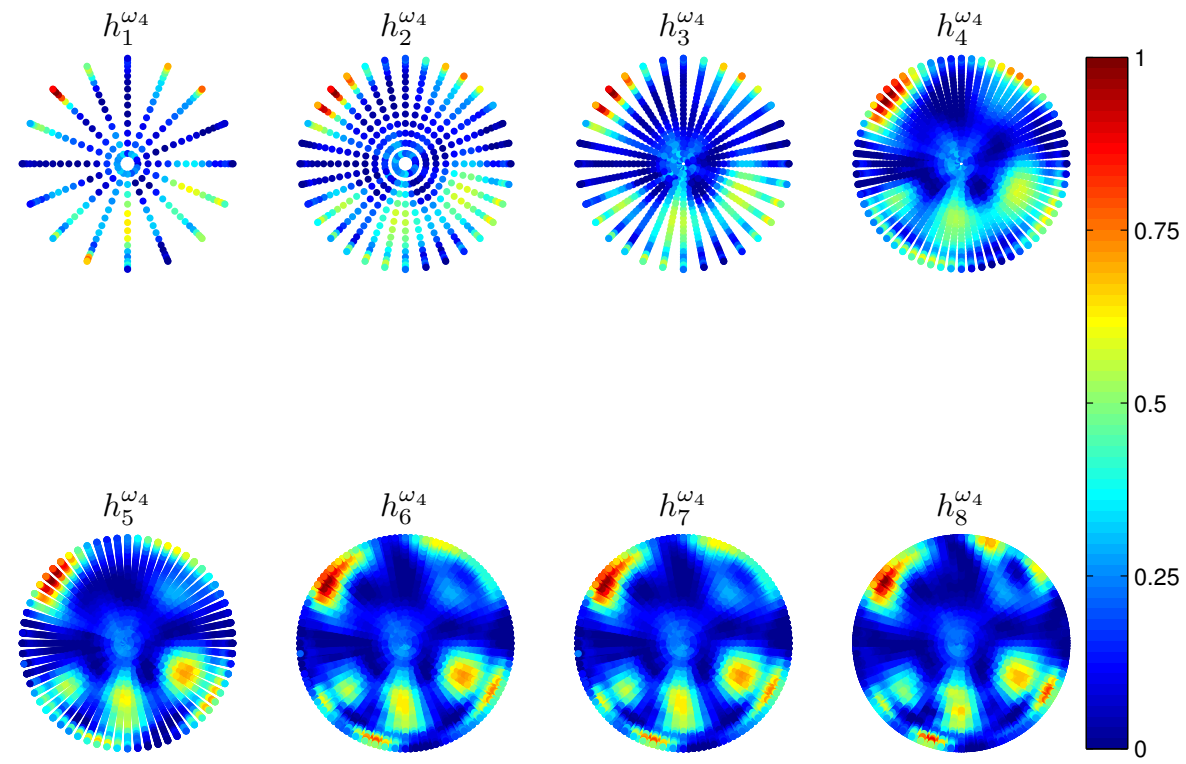

(b) Interpolation results

Figure 17: Directivity polynomial fit over the spheres $S_{R, k}$ for the case 2: harmonic component $\omega_{4}=2 \pi\left(-2 f_{1}+f_{2}\right)$. (a): directivity calculated with BEM $h_{\text {bem, }}^{\omega_{4}} ;($ b): interpolated directivity $h_{k}^{\omega_{4}}$ 

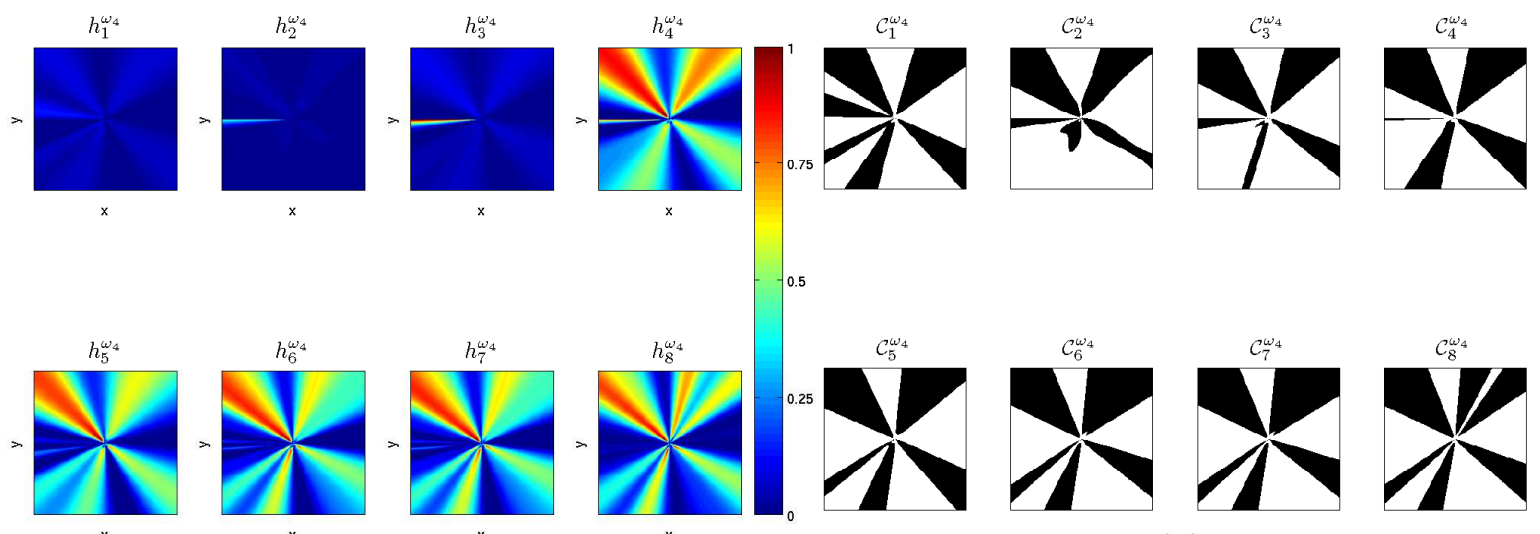

(a)

(b)

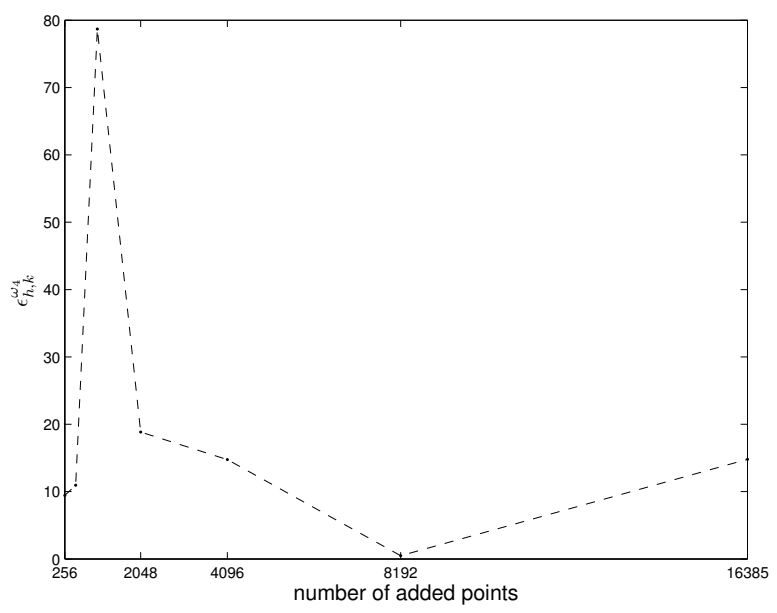

(c)
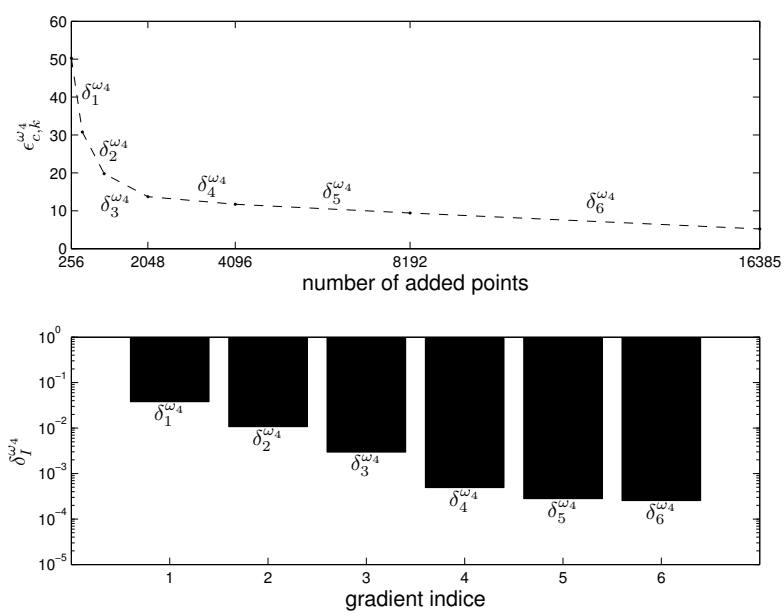

(d)

Figure 18: Directivity convergence with respect to the number of BEM calculation points for the case 2: harmonic component $\omega_{4}=2 \pi\left(-2 f_{1}+f_{2}\right)$. (a): Directivity over the field plane; (b): detected pattern; (c): global error $\epsilon_{h, k}^{\omega_{4}} ;(d)$ : top: pattern error $\epsilon_{C, k}^{\omega_{4}}$ - gradient $\delta_{I}^{\omega_{4}}$; 

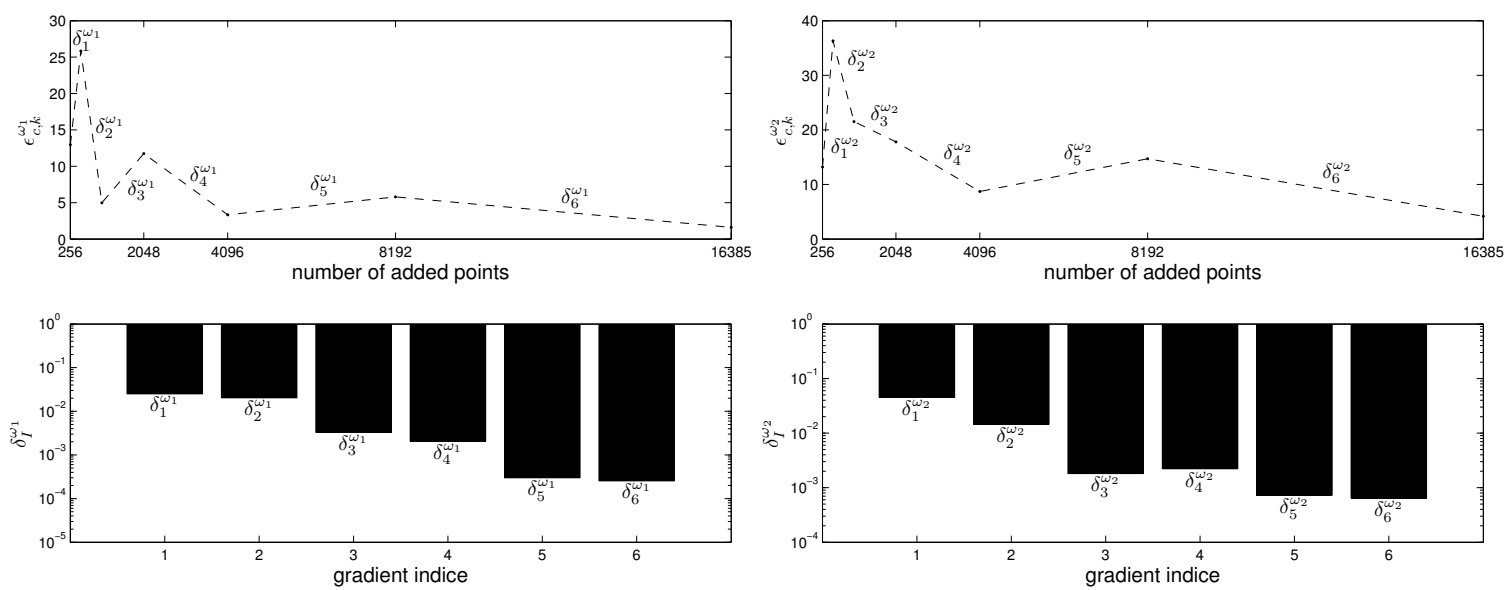

(a)

(b)
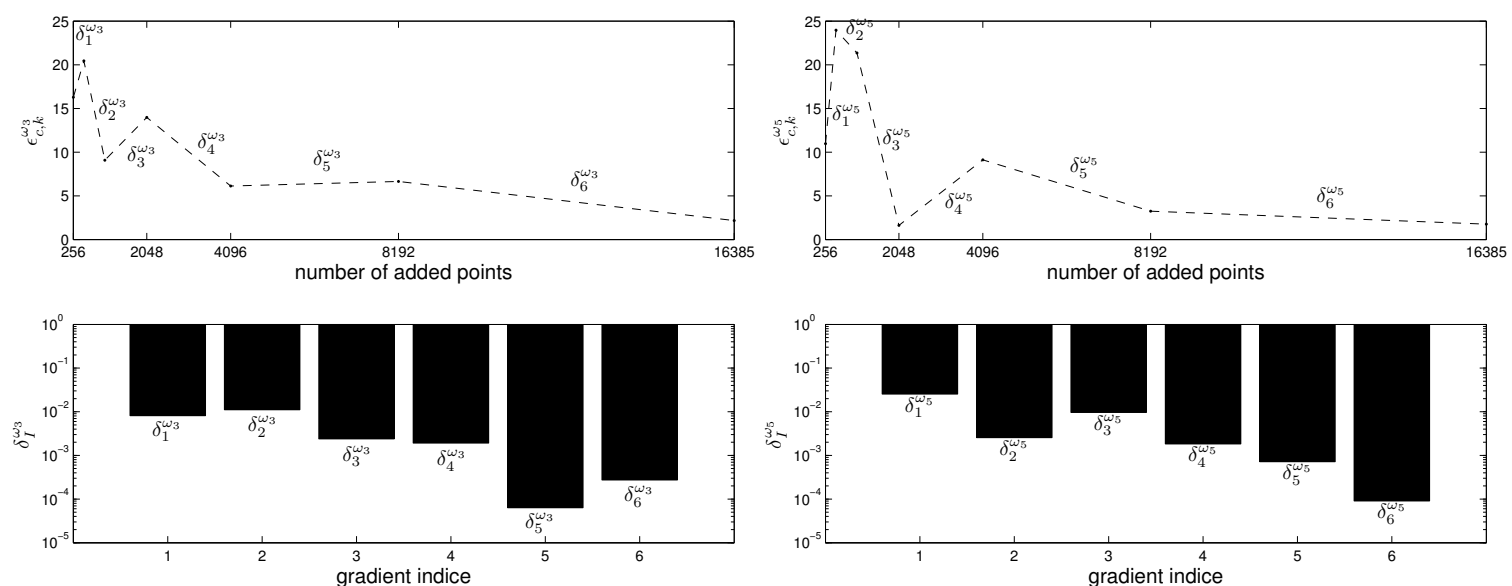

(c)

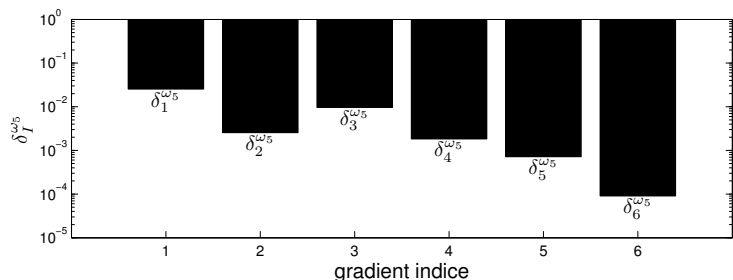

(d)

Figure 19: Directivity pattern relative error $\epsilon_{C, k}^{\omega_{i}}$ for the component (a) $\omega_{1}$, (b) $\omega_{2}$, (c) $\omega_{3}$ and (d) $\omega_{5}$. 


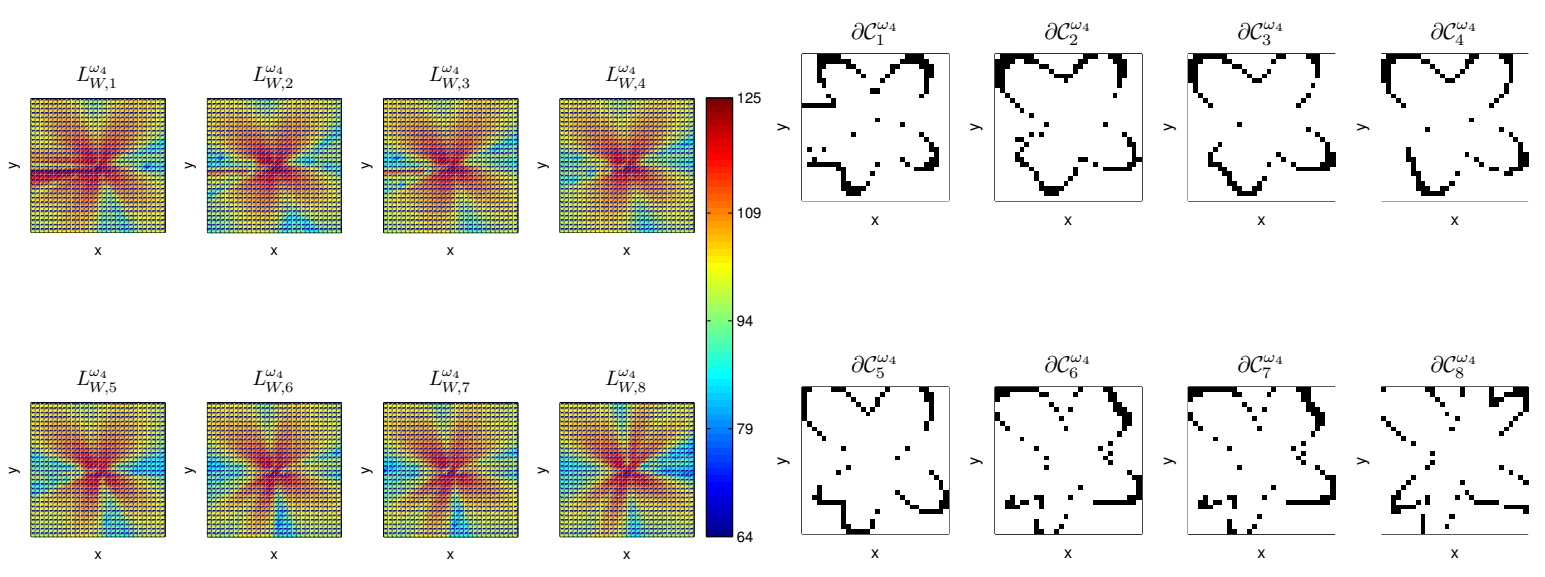

(a)

(b)

Figure 20: Acoustic power levels reconstruction for the case 2 associated with the harmonic component $\omega_{4}=2 \pi\left(-2 f_{1}+f_{2}\right)$. (a): Reconstruction of the approximated acoustic power level $L_{W, k}^{\omega_{4}} ;(b)$ : detected iso-value $\partial \mathscr{C}_{k}^{\omega_{4}}$ 


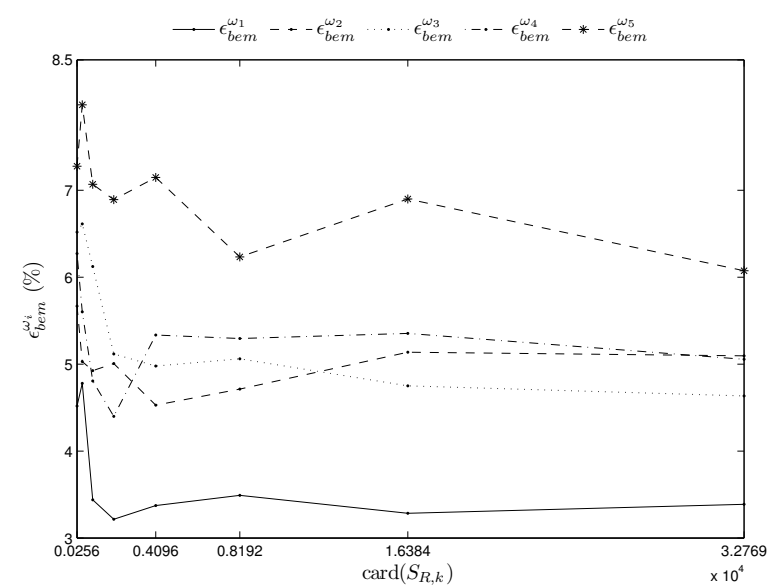

(a)

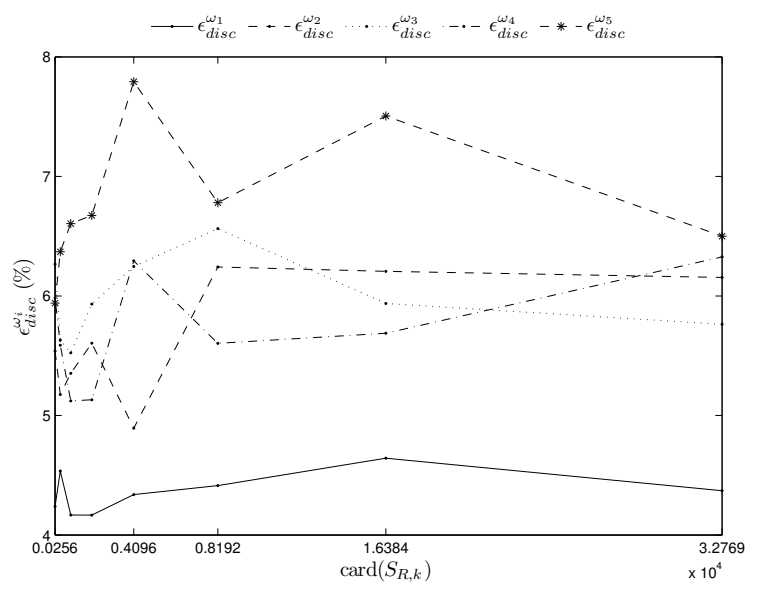

(b)

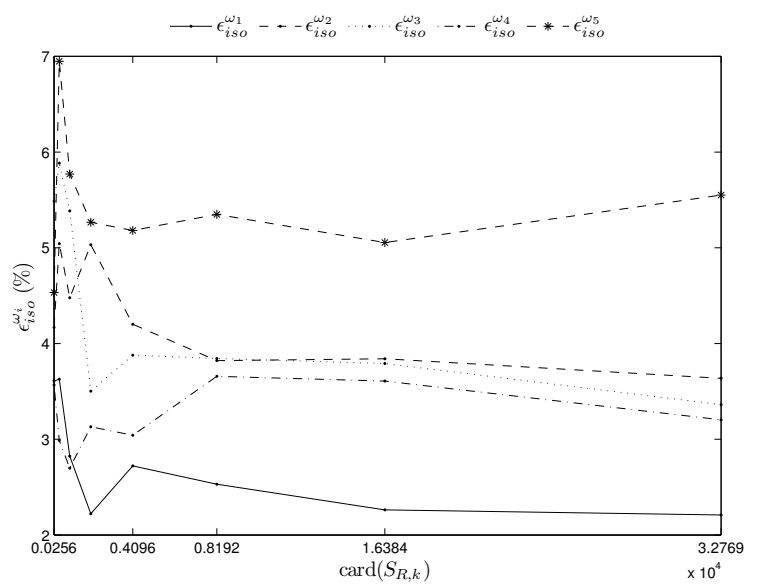

(c)

Figure 21: Estimation of accuracy of the approximated acoustic power levels for the case 2. (a): Comparison with the direct BEM calculation; (b): comparison with the partial BEM: disc; (c): comparison with the partial BEM: iso-value 